Supporting Information:

\title{
Agricultural Plastic Pollution in China: Generation of Plastic Debris and Emission of PAEs from Agricultural Films
}

Qian-Qian Zhang ${ }^{1,2}$, Zhao-Rong Ma ${ }^{1,2}$, Ya-Ya Cai ${ }^{1,2}$, Hui-Ru Li1 ${ }^{1,2}$, Guang-guo Ying ${ }^{1,}$ $2, *$

${ }^{1}$ SCNU Environmental Research Institute, Guangdong Provincial Key Laboratory of Chemical Pollution and Environmental Safety \& MOE Key Laboratory of Theoretical Chemistry of Environment, South China Normal University, Guangzhou 510006, China

${ }^{2}$ School of Environment, South China Normal University, University Town, Guangzhou 510006, China

* Corresponding author. Tel.: +86 020393130796 ;

Email address: guangguo.ying@m.scnu.edu.cn (GGY).

Number of pages: 39

Number of texts: 4

Number of figures: 5

Number of tables: 17 


\section{Text S1. Calculations for greenhouse film use-area and covered area.}

According to the results of field research, plastic tunnel are the main greenhouse in China, which is also the research model of greenhouse film in this study. Based on the publications (Wang et al., 2021), the usable area of film (Sout) were calculated by eq S1.

Sout $=\{\pi \times(H o u t-H s)+2 \times[W / 2-(H o u t-H s)]+2 \times H s\} \times L$

where Hout is the height of outer polytunnel of plastic tunnels (m), $2.48 \mathrm{~m}$; $H_{S}$ is the shoulder height of plastic tunnels $(\mathrm{m}), 1.31 \mathrm{~m}$; W is the width of plastic tunnels $(\mathrm{m})$, $7.79 \mathrm{~m} ; L$ is the length of the plastic tunnels $(\mathrm{m})$. For each of the greenhouse, the $\mathrm{L}$ is $47.63 \mathrm{~m}$. However in our study, each province is abstracted and simplified into a greenhouse. In addition, Wang et al. (2021) also reported the film weight per unit area $\left(D, \mathrm{~kg} / \mathrm{m}^{2}\right)$ for five type of film, and the average value was used in our paper, which is $0.093 \mathrm{~kg} / \mathrm{m}^{2}$. Combing the usage of greenhouse film of each province $(G F$, ton $/ \mathrm{yr})$, the $L$ for of greenhouse in each province were calculated by eq S2.

$L=G F^{*} 1000 / \mathrm{D} /\{\pi \times(H o u t-H s)+2 \times[W / 2-(H o u t-H s)]+2 \times H s\}$

Following, the land covered area of greenhouse film ( $A_{2}$, model parameter, Table S11) is the product of $W$ and $L$. The air area $\left(A_{1}\right.$, model parameter, Table S11) in the greenhouse is same as the Sout.

\section{Text S2. PAEs analysis in agricultural film}

(1) Chemical and materials

A standard mixture of 16 PAEs, including dimethyl-phthalate ester (DMP), diethylphthalate ester (DEP), diisobutyl phthalate (DiBP), dibutyl-phthalate ester (DBP), bis (2-methoxyethyl) phthalate (DMEP), bis(4-methyl-2-pentyl) phthalate (BMPP), bis (2- 
ethoxyethyl) phthalate (DEEP), diamyl phthalate (DPP), Dihexyl phthalate (DHP), benzyl-n-butyl phthalate (BBP), bis(2-n-butoxyethyl) phthalate (DBEP), dicyclohexyl phthalate (DCHP), di-2-ethylhexvl phthalate (DEHP), diphenyl phthalate (DPHP), din-octyl phthalate ester (DOP), and diphenyl phthalate (DNP), and their internal standards 4(di-n-butyl phthalate-3, 4, 5, 6-D4) (DBP-D4) and 4(di (2-ethylhexyl) phthalate-3, 4, 5, 6-D4 (DEHP-D4) were purchased from ANPEL (Shanghai, China) and Sigma-Aldrich (USA). These selected 16 components covered the main kinds of PAEs used in industry manufacture process especially supplied as plasticizers. All the organic solvents (methanol, acetone, dichloromethane, n-hexane, and ethyl acetate) were HPLC grade and purchased from Merck Corporation (Shanghai, China) or CNW Technologies (Dusseldorf, Germany).

(2) Sampling collection and preparation

A total of 12 mulching film samples together with 8 greenhouse film samples were collected from 18 representative provinces all through China, as showed in Table S1. All films were new and unused. The plastic film samples were rinsed clean with deionized water and air dried at ambient temperature. For each of the film, $5 \mathrm{~g}$ sample was weighed, cut into samples pieces $\left(0.25^{*} 0.25 \mathrm{~cm}\right.$ or smaller), and sealed in precleaned aluminum foil bags. All the samples were stored at $-20{ }^{\circ} \mathrm{C}$ prior to sample extraction.

\section{(3) Sample extraction}

For each sample extraction, $0.2 \mathrm{~g}$ of plastic film in a glass centrifuge tube was ultrasonically extracted with $10 \mathrm{~mL}$ ethyl acetate form $15 \mathrm{~min}$. The sample extract was 
transferred to a $50 \mathrm{~mL}$ volumetric flask, then the residue was re-extracted twice. All the three extracts were combined together to a constant volume of $50 \mathrm{~mL}$. A $200 \mathrm{uL}$ mixture was taken out and mixed with $200 \mu \mathrm{L}$ internal standard solution $(1 \mu \mathrm{g} / \mathrm{mL})$ and $600 \mu \mathrm{L}$ ethyl acetate prior to instrumental analysis.

(4) Instrumental analysis

PAEs analysis was performed on an Agilent 7890B gas chromatograph coupled with an Agilent 5977B mass spectrometer (GC-MS) with electron impact ion source. A DB-5MS fused silica capillary column (J\&W Scientific, CA, $30 \mathrm{~m} \times 0.25 \mathrm{~mm}$ i.d., $0.25 \mu \mathrm{m}, \mathrm{USA})$ was installed in $\mathrm{GC}$ with the carrier gas of helium $(1.0 \mathrm{ml} / \mathrm{min})$. The oven temperature started at $80{ }^{\circ} \mathrm{C}(1 \mathrm{~min})$, then ramped to $160{ }^{\circ} \mathrm{C}$ at a rate of $18{ }^{\circ} \mathrm{C}$ $\min ^{-1}$, and continuously to $300{ }^{\circ} \mathrm{C}$ at $9{ }^{\circ} \mathrm{C} \min ^{-1}$ and kept for $5 \mathrm{~min} .1 \mu \mathrm{L}$ sample was injected in splitless mode and selected ion monitoring (SIM) mode. The temperatures for injection port, transfer line, ion source and quadrupole were $280{ }^{\circ} \mathrm{C}, 300{ }^{\circ} \mathrm{C}, 300{ }^{\circ} \mathrm{C}$ and $150{ }^{\circ} \mathrm{C}$, respectively. The detailed monitored ions were listed as in Table S2.

(5) Quality analysis and quality control (QA/QC)

All glass instruments were soaked in chromic acid lotion for $24 \mathrm{~h}$. Centrifuge tubes and titration tubes were washed and dried, and then burned at $450{ }^{\circ} \mathrm{C}$ for $4 \mathrm{~h}$ in muffle furnace. For the glass calorimeter that cannot be burned, it was washed with distilled water, methanol, acetone, dichloromethane and n-hexane in sequence before use. In the course of the experiment, the ordinary pipette was avoided, and the pipette for organic solvent was replaced to avoid the background pollution caused by the plastic gun. We avoided contact with any plastic during the experiment to ensure the validity and 
accuracy of the experimental results. During each set of sample analysed, three lab blank and three standard spiked blank were analysed together with the film samples. None of the analytes were detected in the blank controls. The analytical methods developed for the PAEs showed satisfactory performance in respect of recovery and limit of detection (LOD), as shown in Tables S3. Recoveries of the 15 target PAEs ranged from $83.1 \%$ to $113.9 \%$ with the relative standard deviation (RSD, $n=3$, replicate samples at the same time) $<10 \%$.

\section{Text S3. PAEs emission estimation}

\section{(1) For mulching film.}

I. Mulching time determined. China has a vast territory, and the annual average temperature difference can reach more than 25 degrees (NBSC, 2018). Therefore, the mulching time of plastic film is quite different. Thus the mulching time was firstly set down. Due to the cold and drought conditions in Northwest China, plastic film is required to be covered in the whole growth period of crops, with the longest covering time up to 130 days (Yan CR, 2015). However, in North China, when the potato and corn are planted and uncovered in 60-90 days, the yield and water use efficiency can be improved, and the residue of plastic film can be reduced (Qin L, 2018; Zhao X, 2015). In Southwest China, 70 days is the best time to remove plastic film when fluecured tobacco grows in high altitude area (Yang et al., 1997). Li et al. (2007) found that the best time of uncovering plastic film for potato planting in Fujian Province (South China) is about 45 days, which will neither fail to achieve the expected effect due to 
cold current, nor inhibit crop growth due to excessive soil temperature. In addition, for most crops, when the temperature rises to $15-20^{\circ} \mathrm{C}$, the crops can adapt to the growing environment without plastic film. Therefore, according to the actual temperature in different regions, the suitable film mulching period was selected for subsequent calculation: Northwest China (120 days), Northeast China (120 days), Southwest China (90 days), North China (90 days), East China (60 days), and South China (60 days). Correspondingly, the mulching time in different province were determined and displayed in Table S4.

II. Emission process determined. The emission of DBP and DEHP in mulching film is closely related to its existing state, which can be divided into two stages: during useful lifetime and following useful lifetime, characterized by the recovery rate of agricultural film (Table S5). In other words, the agricultural film is in the "during useful lifetime" stage before recycling, and the "following useful lifetime" stage after recycling. During the mulching film useful lifetime, the PAEs will release into the air and soil at the same time. Zhu et al. (2019) had tested the detailed PAEs emission proportion into air and soil every 15 days in mulching periods from 15 to 150 days. Based on this, we determined the release ratio of PAEs to air and soil in different regions during film covering time combing the mulching time: Northwest China $(63.92 \%$ for air and $16.30 \%$ for soil), Northeast China (63.92\% for air and $16.30 \%$ for soil), Southwest China $(13.58 \%$ for air and $26.27 \%$ for soil), North China (13.58\% for air and $26.27 \%$ for soil), East China (5.09\% for air and $25.67 \%$ for soil), and South China $(5.09 \%$ for air and $25.67 \%$ for soil). After the used mulching film is recycled, the PAEs included in the buried 
residual film would finally release into the soil, and the release amount at this stage is the "following useful life time".

III. Emission estimation equations of PAEs in mulching film. The residual film amount of agricultural film is the amount after the agricultural film is recovered, which can be obtained from the result of Section 2 (Agricultural plastic debris in China) of this paper. In summary, the PAEs emission of mulching film into air compartment $\left(R_{1}\right)$ is from the useful lifetime; the emission into soil compartment $\left(R_{2}\right)$ including the emissions from useful lifetime and emission from residual film. The equations describing the emission estimation are as follows:

$$
\begin{aligned}
& R_{1}=M F \times P A E_{c} \times r_{1} \times 10^{-3} \\
& R_{2}=M F \times P A E_{c} \times r_{2} \times 10^{-3} \\
& R_{3}=M F \times\left(1-f_{r}\right) \times P A E_{c} \times\left(1-r_{1}-r_{2}\right) \times 10^{-3} \\
& R=R_{1}+R_{2}+R_{3}
\end{aligned}
$$

Where $R_{1}$ and $R_{2}$ represent the emission of target chemicals into air and soil, respectively, $\mathrm{kg} / \mathrm{yr}$; $M F$ represents the usage of mulching film of each province, ton/yr, obtained from the China Statistical Yearbook (NBSC, 2018); $P A E_{\mathrm{c}}$ represents contents of target chemicals in mulching film, $\mathrm{g} / \mathrm{ton}$, obtained from the PAEs analysis result in our study above; $f_{\mathrm{r}}$ represents the residual rate for mulching film, obtained from the plastic debris analysis result in our study; $r_{1}$ and $r_{2}$ represent the emission proportion of target pollutants to the air and soil during the useful lifetime, respectively; $R_{3}$ represents the emission of target chemicals into soil from residual mulching film, $\mathrm{kg} / \mathrm{yr} ; R$ represents the emission of target chemicals into environment, $\mathrm{kg} / \mathrm{yr}$. Table S5 lists the 
related parameters needed during the calculation.

\section{(2) For greenhouse film}

I. Emission of PAEs in the greenhouse film determined. When the agricultural film is exposed to the air for 150 days, the release of PAEs can reach $84 \%$ (Zhu et al., 2019). In addition, the release half-lives of DEHP from greenhouse film were tested from 0.5 to 3.2 days (Wang et al., 2021). Considering that the service life of greenhouse in China is generally 2-3 years, the recovery rate of the greenhouse film does not affect the annual emission of PAEs. Combined with these background information, the complete release of PAEs in greenhouse within one year is the basis of the model assumptions. Thus, the amount of PAEs contained in the greenhouse film is the total amount they eventually release, that is the emission of PAEs in the greenhouse film. The total amount of target PAEs was obtained by the content of target chemical in greenhouse film and the amount of greenhouse film in each province of China, as showed in equations below:

$G F=A F-M F$
$R_{g}=G F \times P A E_{g} \times 10^{-3}$

Where $G F$ and $A F$ represent the usage of greenhouse film and mulching film of each province, ton/yr, obtained from the China Statistical Yearbook (NBSC, 2018), and are displayed in Table $\mathrm{S} 5 ; P A E_{\mathrm{g}}$ represents content of target chemical in greenhouse film, $\mathrm{mg} / \mathrm{kg}$, obtained from the PAEs analysis result in our study above; $R_{\mathrm{g}}$ represents the total amount of PAEs in greenhouse film, $\mathrm{kg} / \mathrm{yr}$. 


\section{PAEs emissions of inside and outside greenhouse determined.}

The PAEs included in the greenhouse film would release into inside and outside of the greenhouse. How much do inside and outside receive the PAEs emission is closely related to the temperature (Chen et al., 2018). The temperature inside the greenhouse is suitable for the growth of plants throughout the year (i.e. $20{ }^{\circ} \mathrm{C}-25^{\circ} \mathrm{C}$ ). According to the characteristics of national average temperature and greenhouse inside temperature, and also simplifying the calculation process, the constant value of $20{ }^{\circ} \mathrm{C}$ was selected as the greenhouse inside temperature. However, the temperature outside the greenhouse is the local average temperature. Chen et al. (2018) found that the coefficient of volatile strengths of PAEs, temperature and confinement time of film are related to the volatilization intensity of PAEs, and fit the following equation:

$P A E_{v}=25.48 \times \ln (T+273.15)+1.47 \times \ln t+0.22 \times K-142.88$

Where $P A E_{\mathrm{v}}\left(\mathrm{ug} \cdot \mathrm{m}^{-3}\right)$ is the volatilization intensity; $t$ (day) is the film exposure time, $365 d$ is selected for the its all year round using; $K\left(\mathrm{mg} /\left(\mathrm{g} \cdot \mathrm{m}^{2}\right)\right)$ is the coefficient of volatile strength, and the agricultural film is using the value of $3483.2 ; T\left({ }^{\circ} \mathrm{C}\right)$ is the temperature. For the inside volatilization intensity $\left(P A E_{\mathrm{v} 1}\right)$ calculation, the $T$ is 20 ; while for the outside volatilization intensity $\left(P A E_{\mathrm{v} 2}\right)$, the $\mathrm{T}$ is the local temperature of each province (Table S9). All the calculated volatilization intensity can be found in the Table S7.

PAEs inside and outside the greenhouse together constitute the total PAEs. The emission proportion of total PAEs in the inside and outside greenhouse is determined according to the relative emission intensity of the two. The equations describing the 
emission estimation for inside and outside the greenhouse are as follows:

$$
\begin{aligned}
& R_{g 1}=R_{g} \times \frac{P A E_{v 1}}{P A E_{v 1}+P A E_{v 2}} \\
& R_{g 2}=R_{g} \times \frac{P A E_{v 2}}{P A E_{v 1}+P A E_{v 2}}
\end{aligned}
$$

Where $R_{\mathrm{g} 1}$ and $R_{\mathrm{g} 2}$ represent the emission of target chemicals into inside and outside greenhouse, respectively, ton/yr.

\section{Text S4. Multimedia modelling}

Fugacity $(f, \mathrm{~Pa})$ is a criterion of equilibrium of chemical partition between phases, which is related to concentration $C\left(\mathrm{~mol} / \mathrm{m}^{3}\right)$ by the expression:

$C=f Z$

where $Z$ is the fugacity capacity $\left(\mathrm{mol} / \mathrm{m}^{3} \cdot \mathrm{Pa}\right)$. The function $Z$ depends on the physical and chemical properties of the chemical and various characteristics of each phase, such as temperature and density. For the air compartment, $Z$ is $1 / R T$ for all compounds where $R$ is the gas constant $\left(8.314 \mathrm{~Pa} \cdot \mathrm{m}^{3} / \mathrm{mol} \cdot \mathrm{K}\right)$ and $T$ is the absolute temperature $(\mathrm{K}) . \mathrm{In}$ solid phase, $Z_{\text {solid }}$ equals $K_{\mathrm{OC}} f_{\text {oc }} \rho_{\mathrm{s}} / H$, where $K_{\mathrm{OC}}$ is organic carbon partition coefficient of the solid phase $(\mathrm{L} / \mathrm{kg}) ; f_{\text {oc }}$ is organic carbon fraction of the solid phase and $\rho_{\mathrm{s}}$ is density of the solid phase $(\mathrm{kg} / \mathrm{L})$.

The transfer fluxes $\left(T_{\mathrm{ijk}}\right)(\mathrm{mol} / \mathrm{h})$ could be calculated as $D_{\mathrm{ijk}} f_{\mathrm{i}}$, where $D_{\mathrm{ijk}}$ is a transfer coefficient with unit of $\mathrm{mol} /(\mathrm{h} \cdot \mathrm{Pa})$, and $f_{\mathrm{i}}$ is the fugacity of the compartment with the unit of Pa. Under the steady-state conditions, the total input fluxes from the individual compartments equal to the output fluxes. 
(1) For mulching film. The model treats 2 bulk compartments and 5 sub-compartments: air (air, solid and plant) and soil (air and solid). The environmental processes are expressed by transfer fluxes $(T)$, and in the model include: PAEs emissions to air and soil from mulching film $\left(T_{01 \mathrm{~h}}, T_{02 \mathrm{~h}}\right)$, advective air flows $\left(T_{10 \mathrm{t}}\right)$, degradation in air and soil $\left(T_{10 \mathrm{~m}}, T_{20 \mathrm{~m}}\right)$, accumulation in plant $\left(T_{1 \mathrm{v}}\right)$, diffusion between air and surface soil $\left(T_{12 \mathrm{~d}}\right.$, $\left.T_{21 \mathrm{~d}}\right)$, wet and dry deposition $\left(T_{12 \mathrm{w}}, T_{12 \mathrm{p}}\right)$. The mass balance equation established in terms of transfer fluxes are as follows:

$$
\begin{aligned}
& T_{01 \mathrm{~h}}+T_{21 \mathrm{~d}}=T_{12 \mathrm{~d}}+T_{12 \mathrm{p}}+T_{12 \mathrm{w}}+T_{1 \mathrm{v}}+T_{10 \mathrm{~m}}+T_{10 \mathrm{t}} \\
& T_{02 \mathrm{~h}}+T_{12 \mathrm{~d}}+T_{12 \mathrm{p}}+T_{12 \mathrm{w}}=T_{20 \mathrm{~m}}+T_{21 \mathrm{~d}}
\end{aligned}
$$

Where the subscript number 1 and 2 represent the air and soil compartment, respectively. The definition of the transfer processes and corresponding $D_{\mathrm{ijk}}$ value calculation method are listed in Table S8. Tables S9-S11 list the related parameters needed during the calculation. The transfer fluxes of emission were devised from the result of "PAEs emission estimation". In details, the $T_{01 \mathrm{~h}}$ (unti: $\mathrm{mol} / \mathrm{h}$ ) is the hourly emission converted from $R_{1}$. And the $T_{02 \mathrm{~h}}$ (unti: $\mathrm{mol} / \mathrm{h}$ ) is the hourly emission converted from the summed result of $R_{1}$ and $R_{3}$. The other transfer fluxes can be replaced by some parameters and corresponding calculation methods, as reported in our previous studies (Zhang et al., 2014, 2015a), and the details of the model and related parameters are also described in Tables S8-S11. Those parameters were collected from local Statistical Yearbook, publications and some empirical values from published models. Since the parameter of degradation rate is closely related to temperature, referring to my previous method (Zhang et al., 2015a), the degradation rates at different temperatures were 
calculated in this study. The degradation rate under the experimental conditions from the literature is shown in Table 11-A, and the degradation rate after temperature correction is shown in Table 11-B. Then after collecting values for the parameters used to solve the equations, the model was run in Matlab R2012.

(2) For greenhouse film. The inside greenhouse is treated by using the multimedia model. Plastic tunnel is the basic style for the greenhouse, and the corresponding parameters of the greenhouse shape is referred to Wang et al. (2021), and also described in Text S3. The model framework and parameter description are consistent with the fugacity model of mulching film area. The major difference is that the greenhouse system is a closed system, thus the transfer fluxes of air advection $\left(T_{10 \mathrm{t}}\right)$ and wet deposition from air to soil $\left(T_{12 \mathrm{w}}\right)$ were leaved out. The details of the parameter values are described in Tables S8-S10 and Tables S12. 
Table S1 Details of agricultural film samples.

\begin{tabular}{ccc|ccc}
\hline Sample & Location & Color & Sample & Location & Color \\
\hline MF1 & Beijing & White & MF11 & Gansu & White \\
MF2 & Guangdong & Black & MF12 & Anhui & Black \\
MF3 & Henan & White & GF1 & Jiangsu & White \\
MF4 & Shandong & White & GF2 & Shanxi & Blue \\
MF5 & Gansu & Black & GF3 & Xinjiang & Blue \\
MF6 & Shandong & White & GF4 & Guizhou & White \\
MF7 & Liaoning & White & GF5 & Hebei & White \\
MF8 & Anhui & Black & GF6 & Anhui & White \\
MF9 & Hunan & White & GF7 & Henan & White \\
MF10 & Jiangsu & White & GF8 & Jiangsu & White \\
\hline
\end{tabular}

Note: mulching film: MF; greenhouse film: GF 
Table S2 The detailed monitored ions.

\begin{tabular}{llllc}
\hline Chemicals & abb. & R.T. $^{\text {a }}$ & Qualitative ion & Quantitative ion \\
\hline Dimethyl-phthalate ester & DMP & 7.416 & $163,77,194,133$ & 163 \\
Diethyl-phthalate ester & DEP & 8.753 & $149,177,105,222$ & 149 \\
Diisobutyl phthalate & DiBP & 11.55 & $149,223,104,167$ & 149 \\
Dibutyl-phthalate ester & DBP & 12.532 & $149,223,205,104$ & 149 \\
Bis(2-methoxyethyl) phthalate & DMEP & 12.891 & $149,207,59,104$ & 149 \\
Bis(4-methyl-2-pentyl) phthalate & DMPP & 13.668 & $149,167,85,251$ & 149 \\
Bis(2-ethoxyethyl) phthalate & DEEP & 14.032 & $149,73,104,221$ & 149 \\
Diamyl phthalate & DPP & 14.438 & $149,237,219,104$ & 149 \\
Dihexyl phthalate & DHP & 16.262 & $149,251,233$ & 149 \\
Benzyl-n-butyl phthalate & BBP & 16.359 & $149,91,206,104$ & 149 \\
Bis(2-n-butoxyethyl) phthalate & DBEP & 17.395 & 149,193 & 149 \\
Dicyclohexyl phthalate & DCHP & 17.881 & $149,167,249,104$ & 149 \\
Di-2-ethylhexvl phthalate & DEHP & 17.972 & $149,167,279$ & 149 \\
Diphenyl phthalate & DPhP & 18.103 & $225,77,104,153$ & 225 \\
Di-n-octyl phthalate ester & DOP & 19.595 & $149,279,104,261$ & 149 \\
Diphenyl phthalate & DNP & 21.12 & $149,293,167,275$ & 149 \\
\hline
\end{tabular}

a. Retention time ( $\mathrm{min})$. 
Table S3 Recoveries and limits of detection of the target compounds.

\begin{tabular}{cccc} 
Components of PAEs & Recovery $(\%)$ & RSD $(\%)$ & LOD $\left(\mathrm{mg} \cdot \mathrm{kg}^{-1}\right)$ \\
\hline DMP & 83.29 & 1.77 & 0.0003 \\
DEP & 88.04 & 1.88 & 0.0003 \\
DiBP & 87.85 & 0.87 & 0.0011 \\
DBP & 85.73 & 1.06 & 0.0014 \\
DMEP & 117.91 & 1.20 & 0.0006 \\
BMPP & 94.04 & 1.32 & 0.0006 \\
DEEP & 83.10 & 1.39 & 0.0013 \\
DPP & 109.53 & 2.53 & 0.0016 \\
DHP & 85.89 & 1.86 & 0.0005 \\
BBP & 88.89 & 2.12 & 0.0007 \\
DBEP & 113.91 & 7.68 & 0.0006 \\
DCHP & 91.85 & 0.46 & 0.0015 \\
DEHP & 92.15 & 1.29 & 0.0011 \\
DPHP & 111.35 & 1.79 & 0.0004 \\
DOP & 112.91 & 7.49 & 0.0011 \\
DNP & 104.40 & 4.98 & 0.0003 \\
\hline
\end{tabular}


Table S4 Analytical results of PAEs in different agricultural film samples $\left(\mathrm{mg} \cdot \mathrm{kg}^{-1}\right)$.

\begin{tabular}{|c|c|c|c|c|c|c|c|c|c|c|c|c|c|c|c|c|c|}
\hline Sample & DMP & DEP & DiBP & DBP & BMOP & ВMPP & BEEP & DPP & DHP & BBP & DBEP & DCHP & DEHP & DPHP & DOP & DNP & Total \\
\hline MF1 & ND & ND & 5.40 & 19.95 & ND & ND & ND & ND & ND & ND & ND & ND & 109.71 & ND & ND & ND & 135.06 \\
\hline MF2 & ND & 0.25 & 6.42 & 34.66 & ND & ND & ND & ND & ND & ND & ND & ND & 50.15 & ND & ND & ND & 91.49 \\
\hline MF3 & 0.24 & ND & 3.56 & 41.62 & ND & ND & ND & ND & ND & ND & ND & ND & 45.92 & ND & ND & ND & 91.35 \\
\hline MF4 & 0.08 & ND & 4.71 & 26.03 & ND & ND & ND & ND & ND & ND & ND & ND & 63.60 & ND & ND & ND & 94.42 \\
\hline MF5 & 0.32 & 0.29 & 5.90 & 46.29 & ND & ND & ND & ND & ND & ND & ND & ND & 51.47 & ND & ND & ND & 104.26 \\
\hline MF6 & 0.19 & 0.29 & 3.17 & 13.96 & ND & ND & ND & ND & ND & ND & ND & ND & 42.35 & ND & ND & ND & 59.96 \\
\hline MF7 & ND & 0.15 & 1.20 & 2.94 & ND & ND & ND & ND & ND & ND & ND & ND & 21.38 & ND & ND & ND & 25.65 \\
\hline MF8 & ND & ND & 2.03 & 4.78 & ND & ND & ND & ND & ND & ND & ND & ND & 24.27 & ND & ND & ND & 31.08 \\
\hline MF9 & ND & 0.01 & 2.49 & 8.90 & ND & ND & ND & ND & ND & ND & ND & ND & 57.63 & ND & ND & ND & 69.03 \\
\hline MF10 & ND & ND & 0.99 & 7.93 & ND & ND & ND & ND & ND & ND & ND & ND & 26.63 & ND & ND & ND & 35.56 \\
\hline MF11 & ND & 0.18 & 1.60 & 5.12 & ND & ND & ND & ND & ND & ND & ND & ND & 21.81 & ND & ND & ND & 28.71 \\
\hline MF12 & ND & 0.13 & 1.00 & 29.38 & ND & ND & ND & ND & ND & ND & ND & ND & 27.84 & ND & ND & ND & 58.34 \\
\hline GF1 & ND & ND & 0.52 & 1.81 & ND & ND & ND & ND & ND & ND & ND & ND & 21.70 & ND & ND & ND & 24.03 \\
\hline GF2 & ND & ND & 0.88 & 3.06 & ND & ND & ND & ND & ND & ND & ND & ND & 21.50 & ND & ND & ND & 25.44 \\
\hline GF3 & ND & 0.05 & 0.85 & 8.56 & ND & ND & ND & ND & ND & ND & ND & ND & 21.30 & ND & ND & ND & 30.75 \\
\hline GF4 & 0.24 & ND & 1.27 & 22.40 & ND & ND & ND & $\mathrm{ND}$ & ND & ND & ND & $\mathrm{ND}$ & 23.75 & ND & $\mathrm{ND}$ & ND & 47.66 \\
\hline GF5 & ND & ND & 1.95 & 2.91 & ND & ND & ND & ND & ND & ND & ND & ND & 23.32 & ND & ND & ND & 28.18 \\
\hline GF6 & ND & ND & 0.75 & 2.45 & ND & ND & ND & ND & ND & ND & ND & ND & 21.36 & ND & ND & ND & 24.56 \\
\hline GF7 & ND & ND & ND & 0.33 & ND & ND & ND & ND & ND & ND & ND & ND & 24.81 & ND & ND & ND & 25.14 \\
\hline GF8 & ND & $\mathrm{ND}$ & 0.14 & 2.02 & ND & ND & $\mathrm{ND}$ & ND & ND & ND & ND & ND & 21.82 & ND & ND & ND & 23.98 \\
\hline
\end{tabular}

Note: ND: Not detected. 
Table S5 Parameters for the emission estimation of PAEs for mulching film.

\begin{tabular}{|c|c|c|c|c|c|c|}
\hline \multirow{2}{*}{ Region } & \multirow{2}{*}{ Province } & Mulching time & $A F$ & $M F$ & $r_{1}$ & $r_{2}$ \\
\hline & & (days) & (ton/yr) & (ton/yr) & $(\%)$ & $(\%)$ \\
\hline \multirow{7}{*}{$\begin{array}{c}\text { East } \\
\text { China }\end{array}$} & Shanghai & 60 & 15700 & 3800 & 5.09 & 25.67 \\
\hline & Jiangsu & 60 & 115100 & 44986 & 5.09 & 25.67 \\
\hline & Zhejiang & 60 & 67900 & 28945 & 5.09 & 25.67 \\
\hline & Anhui & 60 & 97600 & 43089 & 5.09 & 25.67 \\
\hline & Fujian & 60 & 62400 & 31882 & 5.09 & 25.67 \\
\hline & Jiangxi & 60 & 53500 & 33198 & 5.09 & 25.67 \\
\hline & Shandong & 60 & 287100 & 114244 & 5.09 & 25.67 \\
\hline \multirow{5}{*}{$\begin{array}{l}\text { South } \\
\text { China }\end{array}$} & Hubei & 60 & 65900 & 37585 & 5.09 & 25.67 \\
\hline & Hunan & 60 & 85200 & 56594 & 5.09 & 25.67 \\
\hline & Guangdong & 60 & 45900 & 26708 & 5.09 & 25.67 \\
\hline & Guangxi & 60 & 47700 & 36300 & 5.09 & 25.67 \\
\hline & Hainan & 60 & 27600 & 16643 & 5.09 & 25.67 \\
\hline \multirow{5}{*}{$\begin{array}{l}\text { North } \\
\text { China }\end{array}$} & Beijing & 90 & 9000 & 2342 & 13.58 & 26.27 \\
\hline & Tianjin & 90 & 10900 & 3751 & 13.58 & 26.27 \\
\hline & Hebei & 90 & 128100 & 61564 & 13.58 & 26.27 \\
\hline & Shanxi & 90 & 50000 & 31734 & 13.58 & 26.27 \\
\hline & Henan & 90 & 157300 & 73023 & 13.58 & 26.27 \\
\hline \multirow{5}{*}{$\begin{array}{c}\text { Northwest } \\
\text { China }\end{array}$} & Shaanxi & 120 & 44000 & 22322 & 63.92 & 16.3 \\
\hline & Gansu & 120 & 172200 & 108388 & 63.92 & 16.3 \\
\hline & Qinghai & 120 & 8400 & 6699 & 63.92 & 16.3 \\
\hline & Ningxia & 120 & 15100 & 11580 & 63.92 & 16.3 \\
\hline & Xinjiang & 120 & 252600 & 219467 & 63.92 & 16.3 \\
\hline \multirow{4}{*}{$\begin{array}{c}\text { Northeast } \\
\text { China }\end{array}$} & Inner Mongolia & 120 & 94300 & 77593 & 63.92 & 16.3 \\
\hline & Liaoning & 120 & 124800 & 37906 & 63.92 & 16.3 \\
\hline & Jilin & 120 & 60800 & 29874 & 63.92 & 16.3 \\
\hline & Heilongjiang & 120 & 79800 & 31185 & 63.92 & 16.3 \\
\hline \multirow{5}{*}{$\begin{array}{c}\text { Southwest } \\
\text { China }\end{array}$} & Chongqing & 90 & 45500 & 24642 & 13.58 & 26.27 \\
\hline & Sichuan & 90 & 131000 & 90949 & 13.58 & 26.27 \\
\hline & Guizhou & 90 & 51100 & 31901 & 13.58 & 26.27 \\
\hline & Yunnan & 90 & 120200 & 96235 & 13.58 & 26.27 \\
\hline & Tibet & 90 & 1900 & 1478 & 13.58 & 26.27 \\
\hline
\end{tabular}


Table S6 The recovery rate of agricultural film in China.

\begin{tabular}{|c|c|c|c|}
\hline \multirow{2}{*}{ Province } & \multicolumn{2}{|c|}{ Recovery rate of agricultural film } & \multirow{2}{*}{ Reference ${ }^{a^{*}}$} \\
\hline & Mulching film & Greenhouse film & \\
\hline Beijing & $86.50 \%$ & $90.50 \%$ & 1 \\
\hline Tianjin & $80.00 \%$ & $90.50 \%$ & 2 \\
\hline Hebei & $67.40 \%$ & $90.50 \%$ & 3 \\
\hline Shanxi & $26.70 \%$ & $90.50 \%$ & 4 \\
\hline Inner Mongolia & $74.50 \%$ & $95.00 \%$ & Note A \\
\hline Liaoning & $95.50 \%$ & $81.80 \%$ & 5 \\
\hline Jilin & $99.10 \%$ & $90.50 \%$ & 6 \\
\hline Heilongjiang & $74.50 \%$ & $90.50 \%$ & Note A \\
\hline Shanghai & $74.50 \%$ & $90.50 \%$ & Note A \\
\hline Jiangsu & $84.30 \%$ & $85.20 \%$ & 7 \\
\hline Zhejiang & $45.00 \%$ & $97.00 \%$ & 8 \\
\hline Anhui & $91.70 \%$ & $84.60 \%$ & 9 \\
\hline Fujian & $80.90 \%$ & $89.50 \%$ & 10 \\
\hline Jiangxi & $74.50 \%$ & $90.50 \%$ & Note A \\
\hline Shandong & $79.00 \%$ & $95.00 \%$ & 11 \\
\hline Henan & $56.30 \%$ & $92.60 \%$ & Note A \\
\hline Hubei & $74.50 \%$ & $90.50 \%$ & Note A \\
\hline Hunan & $74.50 \%$ & $90.50 \%$ & Note A \\
\hline Guangdong & $74.50 \%$ & $90.50 \%$ & Note A \\
\hline Guangxi & $74.50 \%$ & $90.50 \%$ & Note A \\
\hline Hainan & $73.10 \%$ & $90.50 \%$ & 12 \\
\hline Chongqing & $85.20 \%$ & $90.50 \%$ & 13 \\
\hline Sichuan & $75.60 \%$ & $90.50 \%$ & 14 \\
\hline Guizhou & $74.50 \%$ & $90.50 \%$ & Note A \\
\hline Yunnan & $74.50 \%$ & $90.50 \%$ & Note A \\
\hline Tibet & $74.50 \%$ & $90.50 \%$ & Note A \\
\hline Shaanxi & $70.10 \%$ & $90.40 \%$ & 15 \\
\hline Gansu & $63.10 \%$ & $94.40 \%$ & 16 \\
\hline Qinghai & $74.50 \%$ & $90.50 \%$ & Note A \\
\hline Ningxia & $74.50 \%$ & $90.50 \%$ & Note A \\
\hline Xinjiang & $82.00 \%$ & $90.50 \%$ & 17 \\
\hline \multicolumn{4}{|c|}{ a*: 1. https://baijiahao.baidu.com/s?id=1670727280508434499\&wfr=spider\&for=pc } \\
\hline \multicolumn{4}{|c|}{ 2. http://www.mee.gov.cn/ywdt/dfnews/202012/t20201225_814822.shtml } \\
\hline \multicolumn{4}{|c|}{ 3. https://baijiahao.baidu.com/s?id=1674162416667376002\&wfr=spider\&for=pc; } \\
\hline \multicolumn{4}{|c|}{ http://hbrb.hebnews.cn/pc/paper/c/201905/28/c135669.html;http://xxgk.shexian.gov.cn/bmxx/zfxt/xnyncj/201912/t20191225_12 } \\
\hline \multicolumn{4}{|l|}{ 29068.html } \\
\hline \multicolumn{4}{|c|}{ 4. https://www.sohu.com/a/138857634_119038 } \\
\hline \multicolumn{4}{|c|}{ 5. https://baijiahao.baidu.com/s?id=1684688513891814224\&wfr=spider\&for=pc; } \\
\hline \multicolumn{4}{|c|}{ 6. http://k.sina.com.cn/article_1655444627_62ac1493020018jst.html?from=local } \\
\hline https://baijiahao.baid & $3342592136358 \& w$ & & \\
\hline
\end{tabular}


https://baijiahao.baidu.com/s?id=1686510534028367511\&wfr=spider\&for=pc;

https://new.qq.com/omn/20201228/20201228A0IYZY00.html; http://field.10jqka.com.cn/20201228/c625684520.shtml;

http://www.changzhou.gov.cn/ns_news/895160817142796;

http://www.zgjssw.gov.cn/shixianchuanzhen/zhenjiang/201909/t20190903_6320192.shtml;

http://www.agrichem.cn/n/2020/12/10/2013205653.shtml;

https://baijiahao.baidu.com/s?id=1678164030489275823\&wfr=spider\&for=pc;

https://baijiahao.baidu.com/s?id=1672923654287725937\&wfr=spider\&for $=$ pc

8. http://www.wzs.org.cn/hg/201907/t20190709 302534.shtml;

https://z.hangzhou.com.cn/2020/hzrmzfgb/content/content_7866438.html

9. http://sthjt.ah.gov.cn/hbzx/gzdt/sxdt/119426251.html

10. http://sthjj.quanzhou.gov.cn/xxgk/zfxxgkzl/zfxxgkml/tjdcxx/202005/t20200528_2297292.htm;

http://city.ce.cn/news/202012/25/t20201225_7300068.shtml

11. http://www.fx361.com/page/2020/0816/6956957.shtml; http://www.weihai.gov.cn/art/2019/10/5/art_51909_2160970.html

12. https://baijiahao.baidu.com/s?id=1665267419240628530\&wfr=spider\&for=pc

13. https://www.cbg.cn/show/4933-218848.html; https://www.cbg.cn/show/4928-227489.html

14. https://baijiahao.baidu.com/s?id=1657474745563177700\&wfr=spider\&for=pc

15. http://www.sanqin.com/2020-07/13/content_8609752.html; http://www.ankang.gov.cn/content-2132289.html

16. https://www.xianjichina.com/special/detail_350796.html; https://www.chinanews.com/gn/2017/11-14/8375896.shtml;

http://liangzhou.gsjgbz.gov.cn/lzq_zcfg/201504/t20150413_87492.html;

http://www.guazhou.gov.cn/ContentIndex.aspx?id=93583\&

17. https://www.cenews.com.cn/newpos/xf/202011/t20201120_963455.html;

https://baijiahao.baidu.com/s?id=1677993161461363422\&wfr=spider\&for $=\mathrm{pc}$

Note A: No relevant data is found, and the data was assigned according to the national average 
Table 57 The release intensity of PAEs for inside and outside greenhouse film.

\begin{tabular}{|c|c|c|}
\hline Province & $P A E_{\mathrm{v} 1}$ & $P A E_{\mathrm{v} 2}$ \\
\hline Beijing & 8224 & 7948 \\
\hline Tianjin & 8224 & 7948 \\
\hline Hebei & 8224 & 7969 \\
\hline Shanxi & 8224 & 7880 \\
\hline Inner Mongolia & 8224 & 7783 \\
\hline Liaoning & 8224 & 7824 \\
\hline Jilin & 8224 & 7765 \\
\hline Heilongjiang & 8224 & 7717 \\
\hline Shanghai & 8224 & 8038 \\
\hline Jiangsu & 8224 & 8020 \\
\hline Zhejiang & 8224 & 8053 \\
\hline Anhui & 8224 & 8022 \\
\hline Fujian & 8224 & 8127 \\
\hline Jiangxi & 8224 & 8076 \\
\hline Shandong & 8224 & 7987 \\
\hline Henan & 8224 & 8015 \\
\hline Hubei & 8224 & 8027 \\
\hline Hunan & 8224 & 8038 \\
\hline Guangdong & 8224 & 8150 \\
\hline Guangxi & 8224 & 8145 \\
\hline Hainan & 8224 & 8213 \\
\hline Chongqing & 8224 & 8081 \\
\hline Sichuan & 8224 & 8010 \\
\hline Guizhou & 8224 & 7974 \\
\hline Yunnan & 8224 & 7987 \\
\hline Tibet & 8224 & 7836 \\
\hline Shaanxi & 8224 & 7984 \\
\hline Gansu & 8224 & 7790 \\
\hline Qinghai & 8224 & 7747 \\
\hline Ningxia & 8224 & 7867 \\
\hline Xinjiang & 8224 & 7798 \\
\hline
\end{tabular}


$\underline{\text { Table S8 Transfer processes and corresponding } D \text { values. }}$

\begin{tabular}{cccc}
\hline $\begin{array}{c}\text { Symbol } \\
(\mathrm{mol} / \mathrm{h})\end{array}$ & Transfer process & Related $D$ value $(\mathrm{mol} / \mathrm{h} \cdot \mathrm{Pa})$ & $\begin{array}{c}\text { Fugacity } \\
\text { multiplied } \\
(\mathrm{Pa})\end{array}$ \\
\hline$T_{10 \mathrm{t}}$ & Advective flows out of the area & & $f_{1}$ \\
& through air & $Q_{10 \mathrm{t}} \times Z_{1}$ & \\
$T_{01 \mathrm{~h}}$ & Chemical entering air from & -- & \\
& mulching film & -- & -- \\
$T_{02 \mathrm{~h}}$ & Chemical entering soil from & --- & $f_{1}$ \\
$T_{12 \mathrm{~d}}$ & mulching film & $f_{2} /\left(1 /\left(K_{12} \times Z_{11}\right)+L_{2} /\left(B_{1} \times Z_{11}\right)\right)$ & $f_{2}$ \\
$T_{21 \mathrm{~d}}$ & Diffusion from air to soil & $A_{2} /\left(1 /\left(K_{12} \times Z_{11}\right)+L_{2} /\left(B_{1} \times Z_{11}\right)\right)$ & $f_{1}$ \\
$T_{12 \mathrm{p}}$ & Diffusion from soil to air & $A_{2} \times K_{\mathrm{p}} \times X_{12} \times Z_{12}$ & $f_{1}$ \\
$T_{12 \mathrm{w}}$ & Dry deposition from air to soil & $A_{2} \times K_{\mathrm{w}} \times S_{\mathrm{c}} \times X_{12} \times Z_{12}$ & $f_{1}$ \\
$T_{1 \mathrm{v}}$ & Wet deposition from air to soil & $Y_{\mathrm{v}} \times Z_{1 \mathrm{v}} / \rho_{\mathrm{v}}$ & $f_{1}$ \\
$T_{10 \mathrm{~m}}$ & Bioaccumulation in crop & $K_{\mathrm{m} 1} \times A_{1} \times h_{1} \times Z_{1}$ & $f_{2}$ \\
$T_{20 \mathrm{~m}}$ & Degradation in air & $K_{\mathrm{m} 2} \times A_{2} \times h_{2} \times Z_{2}$ & \\
\hline
\end{tabular}


Table S9 Environmental parameters of mulching film modelling for all provinces (Mackay 2001).

\begin{tabular}{cccc}
\hline Symbol & Unit & Definition & Values \\
\hline$h_{1}$ & $\mathrm{~m}$ & Depth of air & 1000 \\
$h_{2}$ & $\mathrm{~m}$ & Thickness of soil & 0.1 \\
$X_{12}$ & $\mathrm{v} / \mathrm{v}$ & Volume fractions of solids in air & $7.2 \times 10^{-12}$ \\
$X_{21}$ & $\mathrm{v} / \mathrm{v}$ & Volume fractions of air in soil & 0.25 \\
$X_{22}$ & $\mathrm{v} / \mathrm{v}$ & Volume fractions of solids in soil & 0.5 \\
$\rho_{22}$ & $\mathrm{t} / \mathrm{m}^{3}$ & Densities of solids in soil & 2.6 \\
$\rho_{\mathrm{v}}$ & $\mathrm{t} / \mathrm{m}^{3}$ & Densities of crop & 0.883 \\
$R$ & $\mathrm{~Pa} \cdot \mathrm{m}^{3} / \mathrm{mol}$ & Universal gas constant & 8.314 \\
$B_{1}$ & $\mathrm{~m} / \mathrm{h}$ & Molecular diffusivity in air & 0.04 \\
$K_{12}$ & $\mathrm{~m} / \mathrm{h}$ & Air-side mass transfer coefficient over soil & 1 \\
$L_{2}$ & $\mathrm{~m}$ & Diffusion path lengths in soil & 0.05 \\
$K_{\mathrm{p}}$ & $\mathrm{m} / \mathrm{h}$ & Dry deposition velocity & 10.8 \\
\hline
\end{tabular}


Table S10 Parameters of target compounds.

\begin{tabular}{|c|c|c|c|c|c|}
\hline Symbol & Unit & Definition & DBP & DEHP & Reference \\
\hline$H_{25}$ & $\mathrm{~Pa} \cdot \mathrm{m}^{3} / \mathrm{mol}$ & Henry's Law constant & 0.133 & 4.4 & Net et al., 2015 \\
\hline$P s_{25}$ & $\mathrm{~Pa}$ & Vapor pressure & $4.73 \times 10^{-3}$ & $3.4 \times 10^{-5}$ & Net et al., 2015 \\
\hline \multirow[t]{2}{*}{$K_{\mathrm{oc}}$} & $\mathrm{L} / \mathrm{kg}$ & Organic carbon normalized & 8138 & 158489 & EC, 2008 \\
\hline & & partition coefficients & & & \\
\hline \multirow[t]{2}{*}{$B C F_{\mathrm{v}}$} & / & Bioconcentration factors for & 0.117 & 0.166 & Mo et al., 2009 \\
\hline & & crop in air & & & \\
\hline
\end{tabular}


Table S11 Environmental parameters in different provinces for mulching film model.

A. Symbol and definition

\begin{tabular}{|c|c|c|c|}
\hline Symbol & Unit & Definition & Reference \\
\hline$A_{1}$ & $\mathrm{~m}^{2}$ & Area of air phase & NBSC, 2018 \\
\hline$A_{2}$ & $\mathrm{~m}^{2}$ & Area of soil phase & NBSC, 2018 \\
\hline$X_{1 \mathrm{v}}$ & $\mathrm{v} / \mathrm{v}$ & Volume fractions of crop in air & NBSC, 2018 \\
\hline $\mathrm{O}_{22}$ & $\mathrm{~m} / \mathrm{m}$ & Fraction of organic carbon in solids in soil & Liu et al., 2016 \\
\hline$Y_{\mathrm{v}}$ & $\mathrm{t} / \mathrm{h}$ & Production of crop & DRS, 2018 \\
\hline$T$ & $\mathrm{~K}$ & Local absolute temperature & NBSC, 2018 \\
\hline$T_{01 \mathrm{~h}}$ & $\mathrm{~mol} / \mathrm{h}$ & DBP emission rate into air & This study, Table S11 \\
\hline$T_{02 \mathrm{~h}}$ & $\mathrm{~mol} / \mathrm{h}$ & DBP emission rate into soil & This study, Table S11 \\
\hline$K_{\mathrm{m} 1}$ & $1 / \mathrm{h}$ & DBP degradation rate in air & Liang et al., 2008 \\
\hline$K_{\mathrm{m} 2}$ & $1 / \mathrm{h}$ & DBP degradation rate in soil & An et al., 1999 \\
\hline$K_{\mathrm{m} 1}^{\prime}$ & $1 / \mathrm{h}$ & DEHP degradation rate in air & Wang et al., 2019 \\
\hline$K_{\mathrm{m} 2}^{\prime}$ & $1 / \mathrm{h}$ & DEHP degradation rate in soil & Madsen et al., 1999 \\
\hline$K_{\mathrm{w}}$ & $\mathrm{m} / \mathrm{h}$ & Rain rate & MWR, 2018 \\
\hline$S_{c}$ & $\mathrm{~m} / \mathrm{h}$ & Annual average wind speed & MWR, 2018 \\
\hline
\end{tabular}




\section{B. Values}

\begin{tabular}{|c|c|c|c|c|c|c|c|c|c|c|c|c|}
\hline Province & $A_{1}$ & $A_{2}$ & $X_{l v}$ & $\mathrm{O}_{22}$ & $Y_{v}$ & $T$ & $K_{m I}$ & $K_{m 2}$ & $K_{m l}^{\prime}$ & $K_{m 2}^{\prime}$ & $K_{w}$ & $S_{c}$ \\
\hline Beijing & $1.68 \mathrm{E}+10$ & $1.16 \mathrm{E}+08$ & $1.70 \mathrm{E}-06$ & $1.08 \mathrm{E}-02$ & $1.99 \mathrm{E}+01$ & $2.89 \mathrm{E}+02$ & 2.17E-02 & $3.47 \mathrm{E}-04$ & $1.93 \mathrm{E}-02$ & $3.36 \mathrm{E}-04$ & $6.76 \mathrm{E}-05$ & $8.46 \mathrm{E}+03$ \\
\hline Tianjin & $1.13 \mathrm{E}+10$ & $5.37 \mathrm{E}+08$ & $1.24 \mathrm{E}-06$ & $1.10 \mathrm{E}-02$ & $6.71 \mathrm{E}+01$ & $2.89 \mathrm{E}+02$ & 2.17E-02 & $3.47 \mathrm{E}-04$ & $1.93 \mathrm{E}-02$ & $3.36 \mathrm{E}-04$ & $5.67 \mathrm{E}-05$ & $1.64 \mathrm{E}+04$ \\
\hline Hebei & $1.88 \mathrm{E}+11$ & $1.00 \mathrm{E}+10$ & $1.20 \mathrm{E}-06$ & 8.97E-03 & $1.21 \mathrm{E}+03$ & $2.90 \mathrm{E}+02$ & 2.29E-02 & $3.67 \mathrm{E}-04$ & $2.04 \mathrm{E}-02$ & $3.36 \mathrm{E}-04$ & $5.47 \mathrm{E}-05$ & $5.94 \mathrm{E}+03$ \\
\hline Shanxi & $1.56 \mathrm{E}+11$ & $5.88 \mathrm{E}+09$ & 7.29E-07 & $7.43 \mathrm{E}-03$ & $4.32 \mathrm{E}+02$ & $2.87 \mathrm{E}+02$ & $1.80 \mathrm{E}-02$ & $2.88 \mathrm{E}-04$ & $1.60 \mathrm{E}-02$ & $3.36 \mathrm{E}-04$ & $6.62 \mathrm{E}-05$ & $8.46 \mathrm{E}+03$ \\
\hline Inner Mongolia & $1.18 \mathrm{E}+12$ & $1.33 \mathrm{E}+10$ & $6.08 \mathrm{E}-07$ & $1.43 \mathrm{E}-02$ & $8.17 \mathrm{E}+02$ & $2.83 \mathrm{E}+02$ & $1.38 \mathrm{E}-02$ & 2.21E-04 & $1.23 \mathrm{E}-02$ & $1.83 \mathrm{E}-04$ & $2.38 \mathrm{E}-05$ & $5.58 \mathrm{E}+03$ \\
\hline Liaoning & $1.46 \mathrm{E}+11$ & $3.11 \mathrm{E}+09$ & $1.17 \mathrm{E}-06$ & $1.08 \mathrm{E}-02$ & $3.66 \mathrm{E}+02$ & $2.84 \mathrm{E}+02$ & $1.54 \mathrm{E}-02$ & $2.47 \mathrm{E}-04$ & $1.38 \mathrm{E}-02$ & $1.83 \mathrm{E}-04$ & $6.21 \mathrm{E}-05$ & $1.08 \mathrm{E}+04$ \\
\hline Jilin & $1.87 \mathrm{E}+11$ & $1.93 \mathrm{E}+09$ & $9.83 \mathrm{E}-07$ & $1.77 \mathrm{E}-02$ & $1.91 \mathrm{E}+02$ & $2.82 \mathrm{E}+02$ & $1.32 \mathrm{E}-02$ & 2.11E-04 & $1.17 \mathrm{E}-02$ & $1.83 \mathrm{E}-04$ & $6.80 \mathrm{E}-05$ & $1.39 \mathrm{E}+04$ \\
\hline Heilongjiang & $4.55 \mathrm{E}+11$ & $2.85 \mathrm{E}+09$ & 7.33E-07 & 2.49E-02 & $2.10 \mathrm{E}+02$ & $2.80 \mathrm{E}+02$ & $1.15 \mathrm{E}-02$ & $1.85 \mathrm{E}-04$ & $1.03 \mathrm{E}-02$ & $1.83 \mathrm{E}-04$ & $6.01 \mathrm{E}-05$ & $1.31 \mathrm{E}+04$ \\
\hline Shanghai & $6.30 \mathrm{E}+09$ & $1.66 \mathrm{E}+08$ & $1.46 \mathrm{E}-06$ & $1.55 \mathrm{E}-02$ & $2.43 \mathrm{E}+01$ & $2.93 \mathrm{E}+02$ & $2.76 \mathrm{E}-02$ & 4.42E-04 & $2.46 \mathrm{E}-02$ & $3.36 \mathrm{E}-04$ & $1.36 \mathrm{E}-04$ & $1.13 \mathrm{E}+04$ \\
\hline Jiangsu & $1.03 \mathrm{E}+11$ & $6.02 \mathrm{E}+09$ & $1.35 \mathrm{E}-06$ & $1.15 \mathrm{E}-02$ & $8.17 \mathrm{E}+02$ & $2.92 \mathrm{E}+02$ & 2.63E-02 & $4.21 \mathrm{E}-04$ & $2.35 \mathrm{E}-02$ & $3.36 \mathrm{E}-04$ & $1.15 \mathrm{E}-04$ & $9.36 \mathrm{E}+03$ \\
\hline Zhejiang & $1.02 \mathrm{E}+11$ & $1.58 \mathrm{E}+09$ & $1.32 \mathrm{E}-06$ & $1.61 \mathrm{E}-02$ & $2.09 \mathrm{E}+02$ & $2.93 \mathrm{E}+02$ & $2.88 \mathrm{E}-02$ & 4.61E-04 & $2.57 \mathrm{E}-02$ & $3.36 \mathrm{E}-04$ & $1.78 \mathrm{E}-04$ & $8.10 \mathrm{E}+03$ \\
\hline Anhui & $1.40 \mathrm{E}+11$ & $4.28 \mathrm{E}+09$ & $8.46 \mathrm{E}-07$ & $1.11 \mathrm{E}-02$ & $3.65 \mathrm{E}+02$ & $2.92 \mathrm{E}+02$ & $2.65 \mathrm{E}-02$ & 4.24E-04 & $2.36 \mathrm{E}-02$ & $3.36 \mathrm{E}-04$ & $1.43 \mathrm{E}-04$ & $9.18 \mathrm{E}+03$ \\
\hline Fujian & $1.21 \mathrm{E}+11$ & $1.40 \mathrm{E}+09$ & $1.30 \mathrm{E}-06$ & $1.57 \mathrm{E}-02$ & $1.83 \mathrm{E}+02$ & $2.96 \mathrm{E}+02$ & $3.52 \mathrm{E}-02$ & $5.63 \mathrm{E}-04$ & $3.14 \mathrm{E}-02$ & $5.55 \mathrm{E}-04$ & $1.73 \mathrm{E}-04$ & $1.01 \mathrm{E}+04$ \\
\hline Jiangxi & $1.67 \mathrm{E}+11$ & $1.34 \mathrm{E}+09$ & 7.87E-07 & $1.60 \mathrm{E}-02$ & $1.06 \mathrm{E}+02$ & $2.94 \mathrm{E}+02$ & $3.07 \mathrm{E}-02$ & 4.90E-04 & $2.73 \mathrm{E}-02$ & $3.36 \mathrm{E}-04$ & $1.89 \mathrm{E}-04$ & $1.17 \mathrm{E}+04$ \\
\hline Shandong & $1.54 \mathrm{E}+11$ & $1.99 \mathrm{E}+10$ & $1.35 \mathrm{E}-06$ & 7.72E-03 & $2.71 \mathrm{E}+03$ & $2.91 \mathrm{E}+02$ & $2.40 \mathrm{E}-02$ & $3.85 \mathrm{E}-04$ & $2.15 \mathrm{E}-02$ & $3.36 \mathrm{E}-04$ & $7.26 \mathrm{E}-05$ & $1.08 \mathrm{E}+04$ \\
\hline
\end{tabular}




\begin{tabular}{|c|c|c|c|c|c|c|c|c|c|c|c|c|}
\hline Province & $A_{1}$ & $A_{2}$ & $X_{l v}$ & $\mathrm{O}_{22}$ & $Y_{v}$ & $T$ & $K_{m l}$ & $K_{m 2}$ & $K_{m l}^{\prime}$ & $K_{m 2}^{\prime}$ & $K_{w}$ & $S_{c}$ \\
\hline Henan & $1.67 \mathrm{E}+11$ & $9.84 \mathrm{E}+09$ & $1.09 \mathrm{E}-06$ & 8.35E-03 & $1.08 \mathrm{E}+03$ & $2.92 \mathrm{E}+02$ & $2.60 \mathrm{E}-02$ & 4.15E-04 & 2.32E-02 & $3.36 \mathrm{E}-04$ & $9.45 \mathrm{E}-05$ & $1.08 \mathrm{E}+04$ \\
\hline Hubei & $1.86 \mathrm{E}+11$ & $4.04 \mathrm{E}+09$ & $9.44 \mathrm{E}-07$ & $1.24 \mathrm{E}-02$ & $3.84 \mathrm{E}+02$ & $2.92 \mathrm{E}+02$ & $2.69 \mathrm{E}-02$ & 4.30E-04 & $2.40 \mathrm{E}-02$ & $3.36 \mathrm{E}-04$ & $1.49 \mathrm{E}-04$ & $9.54 \mathrm{E}+03$ \\
\hline Hunan & $2.12 \mathrm{E}+11$ & $7.24 \mathrm{E}+09$ & $9.22 \mathrm{E}-07$ & $1.91 \mathrm{E}-02$ & $6.73 \mathrm{E}+02$ & $2.93 \mathrm{E}+02$ & $2.76 \mathrm{E}-02$ & 4.42E-04 & $2.46 \mathrm{E}-02$ & $3.36 \mathrm{E}-04$ & $1.71 \mathrm{E}-04$ & $9.72 \mathrm{E}+03$ \\
\hline Guangdong & $1.80 \mathrm{E}+11$ & $1.39 \mathrm{E}+09$ & $1.10 \mathrm{E}-06$ & $1.42 \mathrm{E}-02$ & $1.55 \mathrm{E}+02$ & $2.97 \mathrm{E}+02$ & $3.75 \mathrm{E}-02$ & $6.00 \mathrm{E}-04$ & $3.34 \mathrm{E}-02$ & $5.55 \mathrm{E}-04$ & $1.99 \mathrm{E}-04$ & $7.56 \mathrm{E}+03$ \\
\hline Guangxi & $2.36 \mathrm{E}+11$ & $5.75 \mathrm{E}+09$ & $8.35 \mathrm{E}-07$ & $1.75 \mathrm{E}-02$ & $4.84 \mathrm{E}+02$ & $2.97 \mathrm{E}+02$ & $3.70 \mathrm{E}-02$ & $5.91 \mathrm{E}-04$ & $3.30 \mathrm{E}-02$ & $5.55 \mathrm{E}-04$ & $2.06 \mathrm{E}-04$ & $6.12 \mathrm{E}+03$ \\
\hline Hainan & $3.40 \mathrm{E}+10$ & $4.87 \mathrm{E}+08$ & $1.01 \mathrm{E}-06$ & $9.52 \mathrm{E}-03$ & $4.94 \mathrm{E}+01$ & $3.00 \mathrm{E}+02$ & $4.46 \mathrm{E}-02$ & 7.13E-04 & $3.98 \mathrm{E}-02$ & $5.55 \mathrm{E}-04$ & $2.35 \mathrm{E}-04$ & $1.12 \mathrm{E}+04$ \\
\hline Chongqing & $8.23 \mathrm{E}+10$ & $2.57 \mathrm{E}+09$ & $9.70 \mathrm{E}-07$ & $1.20 \mathrm{E}-02$ & $2.51 \mathrm{E}+02$ & $2.95 \mathrm{E}+02$ & $3.11 \mathrm{E}-02$ & 4.97E-04 & $2.77 \mathrm{E}-02$ & $3.36 \mathrm{E}-04$ & $1.46 \mathrm{E}-04$ & $4.68 \mathrm{E}+03$ \\
\hline Sichuan & $4.81 \mathrm{E}+11$ & $9.97 \mathrm{E}+09$ & $9.18 \mathrm{E}-07$ & $1.59 \mathrm{E}-02$ & $9.22 \mathrm{E}+02$ & $2.92 \mathrm{E}+02$ & $2.56 \mathrm{E}-02$ & 4.10E-04 & $2.28 \mathrm{E}-02$ & $3.36 \mathrm{E}-04$ & 1.07E-04 & $3.60 \mathrm{E}+03$ \\
\hline Guizhou & $1.76 \mathrm{E}+11$ & $3.20 \mathrm{E}+09$ & $6.80 \mathrm{E}-07$ & $2.10 \mathrm{E}-02$ & $2.20 \mathrm{E}+02$ & $2.90 \mathrm{E}+02$ & 2.32E-02 & $3.72 \mathrm{E}-04$ & $2.07 \mathrm{E}-02$ & $3.36 \mathrm{E}-04$ & $1.34 \mathrm{E}-04$ & $7.56 \mathrm{E}+03$ \\
\hline Yunnan & $3.83 \mathrm{E}+11$ & $1.07 \mathrm{E}+10$ & $6.60 \mathrm{E}-07$ & $1.93 \mathrm{E}-02$ & $7.09 \mathrm{E}+02$ & $2.91 \mathrm{E}+02$ & $2.40 \mathrm{E}-02$ & $3.85 \mathrm{E}-04$ & $2.15 \mathrm{E}-02$ & $3.36 \mathrm{E}-04$ & $1.54 \mathrm{E}-04$ & $7.74 \mathrm{E}+03$ \\
\hline Tibet & $1.23 \mathrm{E}+12$ & $3.18 \mathrm{E}+07$ & $8.42 \mathrm{E}-07$ & $1.66 \mathrm{E}-02$ & $2.69 \mathrm{E}+00$ & $2.85 \mathrm{E}+02$ & $1.60 \mathrm{E}-02$ & $2.56 \mathrm{E}-04$ & $1.43 \mathrm{E}-02$ & $1.83 \mathrm{E}-04$ & $7.21 \mathrm{E}-05$ & $7.20 \mathrm{E}+03$ \\
\hline Shaanxi & $2.06 \mathrm{E}+11$ & $4.37 \mathrm{E}+09$ & $8.05 \mathrm{E}-07$ & $8.51 \mathrm{E}-03$ & $3.55 \mathrm{E}+02$ & $2.91 \mathrm{E}+02$ & 2.39E-02 & $3.82 \mathrm{E}-04$ & $2.13 \mathrm{E}-02$ & $3.36 \mathrm{E}-04$ & $9.15 \mathrm{E}-05$ & $7.20 \mathrm{E}+03$ \\
\hline Gansu & $4.54 \mathrm{E}+11$ & $1.39 \mathrm{E}+10$ & $7.08 \mathrm{E}-07$ & $9.79 \mathrm{E}-03$ & $9.91 \mathrm{E}+02$ & $2.83 \mathrm{E}+02$ & $1.41 \mathrm{E}-02$ & $2.26 \mathrm{E}-04$ & $1.26 \mathrm{E}-02$ & $1.83 \mathrm{E}-04$ & 3.63E-05 & $3.24 \mathrm{E}+03$ \\
\hline Qinghai & $7.22 \mathrm{E}+11$ & $7.64 \mathrm{E}+08$ & $5.05 \mathrm{E}-07$ & $1.41 \mathrm{E}-02$ & $3.88 \mathrm{E}+01$ & $2.81 \mathrm{E}+02$ & $1.25 \mathrm{E}-02$ & $2.01 \mathrm{E}-04$ & $1.12 \mathrm{E}-02$ & $1.83 \mathrm{E}-04$ & $3.87 \mathrm{E}-05$ & $6.48 \mathrm{E}+03$ \\
\hline Ningxia & $6.64 \mathrm{E}+10$ & $2.00 \mathrm{E}+09$ & $8.98 \mathrm{E}-07$ & $7.04 \mathrm{E}-03$ & $1.81 \mathrm{E}+02$ & $2.86 \mathrm{E}+02$ & $1.74 \mathrm{E}-02$ & $2.78 \mathrm{E}-04$ & $1.55 \mathrm{E}-02$ & $3.36 \mathrm{E}-04$ & $3.79 \mathrm{E}-05$ & $6.12 \mathrm{E}+03$ \\
\hline Xinjiang & $1.66 \mathrm{E}+12$ & $3.80 \mathrm{E}+10$ & $6.37 \mathrm{E}-07$ & $9.81 \mathrm{E}-03$ & $2.44 \mathrm{E}+03$ & $2.83 \mathrm{E}+02$ & $1.44 \mathrm{E}-02$ & $2.30 \mathrm{E}-04$ & $1.28 \mathrm{E}-02$ & $1.83 \mathrm{E}-04$ & $2.20 \mathrm{E}-05$ & $8.64 \mathrm{E}+03$ \\
\hline
\end{tabular}


Table S12 Environmental parameters in different provinces for greenhouse film model. For the parameters same as the those in mulching film modelling are not included.

\section{A. Symbol and definition}

\begin{tabular}{cccc}
\hline Symbol & Unit & Definition & Reference \\
\hline$A_{1}$ & $\mathrm{~m}^{2}$ & Area of air phase & Text $\mathrm{S}$ 1 of this paper \\
$A_{2}$ & $\mathrm{~m}^{2}$ & Area of soil phase & Text $\mathrm{S} 1$ of this paper \\
$X_{1 \mathrm{v}}$ & $\mathrm{v} / \mathrm{v}$ & Volume fractions of crop in air & NBSC, 2018 \\
$O_{22}$ & $\mathrm{~m} / \mathrm{m}$ & Fraction of organic carbon in solids in soil & Liu et al., 2016 \\
$Y_{\mathrm{v}}$ & $\mathrm{t} / \mathrm{h}$ & Production of crop & DRS, 2018 \\
$T_{01 \mathrm{~h}}$ & $\mathrm{~mol} / \mathrm{h}$ & DBP emission rate inside greenhouse & Chen et al., 2018 \\
$T^{\prime}{ }_{01 \mathrm{~h}}$ & $\mathrm{~mol} / \mathrm{h}$ & DEHP emission rate inside greenhouse & Chen et al., 2018 \\
\hline
\end{tabular}


B. Values

\begin{tabular}{|c|c|c|c|c|c|c|c|}
\hline Province & $A_{1}$ & $A_{2}$ & $X_{1 \mathrm{v}}$ & $\mathrm{O}_{22}$ & $Y_{\mathrm{v}}$ & $T_{01 \mathrm{~h}}$ & $T_{01 \mathrm{~h}}^{\prime}$ \\
\hline Beijing & $6.95 \mathrm{E}+07$ & $3.77 \mathrm{E}+07$ & $9.94 \mathrm{E}-03$ & $1.08 \mathrm{E}-02$ & $5.46 \mathrm{E}+01$ & 7.52E-01 & $2.21 \mathrm{E}+00$ \\
\hline Tianjin & $7.50 \mathrm{E}+07$ & $4.07 \mathrm{E}+07$ & $5.49 \mathrm{E}-03$ & $1.10 \mathrm{E}-02$ & $9.39 \mathrm{E}+01$ & $8.12 \mathrm{E}-01$ & $2.39 \mathrm{E}+00$ \\
\hline Hebei & $6.98 \mathrm{E}+08$ & $3.78 \mathrm{E}+08$ & $9.76 \mathrm{E}-03$ & 8.97E-03 & $1.76 \mathrm{E}+03$ & $7.54 \mathrm{E}+00$ & $2.22 \mathrm{E}+01$ \\
\hline Shanxi & $1.91 \mathrm{E}+08$ & $1.04 \mathrm{E}+08$ & 4.97E-03 & 7.43E-03 & $2.81 \mathrm{E}+02$ & $2.08 \mathrm{E}+00$ & $6.12 \mathrm{E}+00$ \\
\hline Inner Mongolia & $1.75 \mathrm{E}+08$ & $9.50 \mathrm{E}+07$ & 8.07E-03 & $1.43 \mathrm{E}-02$ & $3.87 \mathrm{E}+02$ & $1.92 \mathrm{E}+00$ & $5.63 \mathrm{E}+00$ \\
\hline Liaoning & $9.11 \mathrm{E}+08$ & $4.94 \mathrm{E}+08$ & $5.26 \mathrm{E}-03$ & $1.08 \mathrm{E}-02$ & $6.26 \mathrm{E}+02$ & $9.94 \mathrm{E}+00$ & $2.92 \mathrm{E}+01$ \\
\hline Jilin & $3.24 \mathrm{E}+08$ & $1.76 \mathrm{E}+08$ & $3.73 \mathrm{E}-03$ & $1.77 \mathrm{E}-02$ & $1.24 \mathrm{E}+02$ & $3.54 \mathrm{E}+00$ & $1.04 \mathrm{E}+01$ \\
\hline Heilongjiang & $5.09 \mathrm{E}+08$ & $2.76 \mathrm{E}+08$ & $3.75 \mathrm{E}-03$ & $2.49 \mathrm{E}-02$ & $2.78 \mathrm{E}+02$ & $5.59 \mathrm{E}+00$ & $1.64 \mathrm{E}+01$ \\
\hline Shanghai & $1.24 \mathrm{E}+08$ & $6.75 \mathrm{E}+07$ & $1.18 \mathrm{E}-02$ & $1.55 \mathrm{E}-02$ & $1.02 \mathrm{E}+02$ & $1.34 \mathrm{E}+00$ & $3.94 \mathrm{E}+00$ \\
\hline Jiangsu & $7.35 \mathrm{E}+08$ & $3.99 \mathrm{E}+08$ & $1.23 \mathrm{E}-02$ & $1.15 \mathrm{E}-02$ & $1.93 \mathrm{E}+03$ & $7.92 \mathrm{E}+00$ & $2.33 \mathrm{E}+01$ \\
\hline Zhejiang & $4.08 \mathrm{E}+08$ & $2.21 \mathrm{E}+08$ & $1.87 \mathrm{E}-02$ & $1.61 \mathrm{E}-02$ & $6.65 \mathrm{E}+02$ & $4.39 \mathrm{E}+00$ & $1.29 \mathrm{E}+01$ \\
\hline Anhui & $5.72 \mathrm{E}+08$ & $3.10 \mathrm{E}+08$ & $7.79 \mathrm{E}-03$ & $1.11 \mathrm{E}-02$ & $7.03 \mathrm{E}+02$ & $6.16 \mathrm{E}+00$ & $1.81 \mathrm{E}+01$ \\
\hline Fujian & $3.20 \mathrm{E}+08$ & $1.74 \mathrm{E}+08$ & $2.20 \mathrm{E}-02$ & $1.57 \mathrm{E}-02$ & $4.93 \mathrm{E}+02$ & $3.43 \mathrm{E}+00$ & $1.01 \mathrm{E}+01$ \\
\hline Jiangxi & $2.13 \mathrm{E}+08$ & $1.15 \mathrm{E}+08$ & $3.80 \mathrm{E}-02$ & $1.60 \mathrm{E}-02$ & $5.19 \mathrm{E}+02$ & $2.29 \mathrm{E}+00$ & $6.72 \mathrm{E}+00$ \\
\hline Shandong & $1.81 \mathrm{E}+09$ & $9.83 \mathrm{E}+08$ & $5.64 \mathrm{E}-03$ & 7.72E-03 & $2.83 \mathrm{E}+03$ & $1.96 \mathrm{E}+01$ & $5.75 \mathrm{E}+01$ \\
\hline Henan & $8.84 \mathrm{E}+08$ & $4.79 \mathrm{E}+08$ & $1.38 \mathrm{E}-02$ & $8.35 \mathrm{E}-03$ & $2.62 \mathrm{E}+03$ & $9.52 \mathrm{E}+00$ & $2.80 \mathrm{E}+01$ \\
\hline Hubei & $2.97 \mathrm{E}+08$ & $1.61 \mathrm{E}+08$ & $2.62 \mathrm{E}-02$ & $1.24 \mathrm{E}-02$ & $1.33 \mathrm{E}+03$ & $3.19 \mathrm{E}+00$ & $9.39 \mathrm{E}+00$ \\
\hline Hunan & $3.00 \mathrm{E}+08$ & $1.63 \mathrm{E}+08$ & $2.09 \mathrm{E}-02$ & $1.91 \mathrm{E}-02$ & $1.28 \mathrm{E}+03$ & $3.23 \mathrm{E}+00$ & $9.49 \mathrm{E}+00$ \\
\hline Guangdong & $2.01 \mathrm{E}+08$ & $1.09 \mathrm{E}+08$ & $6.64 \mathrm{E}-02$ & $1.42 \mathrm{E}-02$ & $1.11 \mathrm{E}+03$ & $2.15 \mathrm{E}+00$ & $6.31 \mathrm{E}+00$ \\
\hline Guangxi & $1.19 \mathrm{E}+08$ & $6.48 \mathrm{E}+07$ & $3.80 \mathrm{E}-02$ & $1.75 \mathrm{E}-02$ & $1.14 \mathrm{E}+03$ & $1.28 \mathrm{E}+00$ & $3.75 \mathrm{E}+00$ \\
\hline Hainan & $1.15 \mathrm{E}+08$ & $6.25 \mathrm{E}+07$ & $3.58 \mathrm{E}-02$ & $9.52 \mathrm{E}-03$ & $1.93 \mathrm{E}+02$ & $1.23 \mathrm{E}+00$ & $3.61 \mathrm{E}+00$ \\
\hline Chongqing & $2.18 \mathrm{E}+08$ & $1.18 \mathrm{E}+08$ & $1.79 \mathrm{E}-02$ & $1.20 \mathrm{E}-02$ & $6.49 \mathrm{E}+02$ & $2.35 \mathrm{E}+00$ & $6.89 \mathrm{E}+00$ \\
\hline Sichuan & $4.20 \mathrm{E}+08$ & $2.28 \mathrm{E}+08$ & $2.02 \mathrm{E}-02$ & $1.59 \mathrm{E}-02$ & $1.48 \mathrm{E}+03$ & $4.53 \mathrm{E}+00$ & $1.33 \mathrm{E}+01$ \\
\hline Guizhou & $2.02 \mathrm{E}+08$ & $1.09 \mathrm{E}+08$ & $2.45 \mathrm{E}-02$ & $2.10 \mathrm{E}-02$ & $7.91 \mathrm{E}+02$ & $2.18 \mathrm{E}+00$ & $6.41 \mathrm{E}+00$ \\
\hline Yunnan & $2.51 \mathrm{E}+08$ & $1.36 \mathrm{E}+08$ & $1.64 \mathrm{E}-02$ & $1.93 \mathrm{E}-02$ & $7.23 \mathrm{E}+02$ & $2.71 \mathrm{E}+00$ & $7.96 \mathrm{E}+00$ \\
\hline Tibet & $4.11 \mathrm{E}+06$ & $2.23 \mathrm{E}+06$ & $1.80 \mathrm{E}-01$ & $1.66 \mathrm{E}-02$ & $2.53 \mathrm{E}+01$ & $4.48 \mathrm{E}-02$ & $1.32 \mathrm{E}-01$ \\
\hline Shaanxi & $2.27 \mathrm{E}+08$ & $1.23 \mathrm{E}+08$ & $8.54 \mathrm{E}-03$ & $8.51 \mathrm{E}-03$ & $6.04 \mathrm{E}+02$ & $2.45 \mathrm{E}+00$ & $7.20 \mathrm{E}+00$ \\
\hline
\end{tabular}




\begin{tabular}{cccccccc}
\hline Province & $A_{1}$ & $A_{2}$ & $X_{1 \mathrm{v}}$ & $O_{22}$ & $Y_{\mathrm{v}}$ & $T_{01 \mathrm{~h}}$ & $T_{01 \mathrm{~h}}^{\prime}$ \\
\hline Gansu & $6.69 \mathrm{E}+08$ & $3.63 \mathrm{E}+08$ & $3.09 \mathrm{E}-03$ & $9.79 \mathrm{E}-03$ & $4.22 \mathrm{E}+02$ & $7.31 \mathrm{E}+00$ & $2.15 \mathrm{E}+01$ \\
Qinghai & $1.80 \mathrm{E}+07$ & $9.76 \mathrm{E}+06$ & $1.58 \mathrm{E}-02$ & $1.41 \mathrm{E}-02$ & $5.16 \mathrm{E}+01$ & $1.97 \mathrm{E}-01$ & $5.80 \mathrm{E}-01$ \\
Ningxia & $3.68 \mathrm{E}+07$ & $1.99 \mathrm{E}+07$ & $1.86 \mathrm{E}-02$ & $7.04 \mathrm{E}-03$ & $1.88 \mathrm{E}+02$ & $4.00 \mathrm{E}-01$ & $1.18 \mathrm{E}+00$ \\
Xinjiang & $3.48 \mathrm{E}+08$ & $1.89 \mathrm{E}+08$ & $6.61 \mathrm{E}-03$ & $9.81 \mathrm{E}-03$ & $6.34 \mathrm{E}+02$ & $3.80 \mathrm{E}+00$ & $1.12 \mathrm{E}+01$ \\
\hline
\end{tabular}


Table S13 The amount of agricultural plastic debris and erosion in China.

\begin{tabular}{|c|c|c|c|c|}
\hline Province/Items & $\begin{array}{l}\text { Amount of } \\
\text { agricultural film } \\
\text { recovered (ton) }\end{array}$ & $\begin{array}{l}\text { Soil erosion of } \\
\text { plastic film } \\
\text { debris (ton) }\end{array}$ & $\begin{array}{c}\text { Residue of } \\
\text { agricultural film } \\
\text { (ton) }\end{array}$ & $\begin{array}{l}\text { Accumulated residue } \\
\text { of agricultural film } \\
\text { (ton) }^{\#}\end{array}$ \\
\hline Beijing & 945 & 8.08 & 937 & $-*$ \\
\hline Tianjin & 1426 & 6.42 & 1419 & $-*$ \\
\hline Hebei & 26358 & 164 & 26193 & 15800 \\
\hline Shanxi & 25000 & 490 & 24510 & 52300 \\
\hline Inner Mongolia & 20608 & 158 & 20450 & 157300 \\
\hline Liaoning & 17509 & 202 & 17307 & $-^{*}$ \\
\hline Jilin & 3204 & 39.4 & 3165 & 8400 \\
\hline Heilongjiang & 12541 & 142 & 12399 & 1500 \\
\hline Shanghai & 2093 & 0.00 & 2093 & 10 \\
\hline Jiangsu & 17462 & 16.2 & 17446 & 4000 \\
\hline Zhejiang & 17088 & 44.7 & 17044 & 123 \\
\hline Anhui & 11971 & 22.3 & 11949 & 4400 \\
\hline Fujian & 9282 & 61.0 & 9221 & 1800 \\
\hline Jiangxi & 10378 & 35.4 & 10343 & $-^{*}$ \\
\hline Shandong & 32634 & 222 & 32412 & $-^{*}$ \\
\hline Henan & 38148 & 193 & 37954 & 11400 \\
\hline Hubei & 12253 & 57.6 & 12196 & 5820 \\
\hline Hunan & 17125 & 56.8 & 17068 & 27200 \\
\hline Guangdong & 8620 & 34.7 & 8585 & 3100 \\
\hline Guangxi & 10327 & 41.5 & 10286 & $-^{*}$ \\
\hline Hainan & 5520 & 20.0 & 5500 & 4333 \\
\hline Chongqing & 5624 & 71.9 & 5552 & 2805 \\
\hline Sichuan & 25938 & 212 & 25726 & 6300 \\
\hline Guizhou & 9944 & 146 & 9798 & 14200 \\
\hline Yunnan & 26788 & 327 & 26461 & $-^{*}$ \\
\hline Tibet & 417 & 2.68 & 414 & $-^{*}$ \\
\hline Shaanxi & 8759 & 199 & 8560 & 13700 \\
\hline Gansu & 43597 & 1260 & 42337 & 78600 \\
\hline Qinghai & 1868 & 14.1 & 1854 & 4482 \\
\hline Ningxia & 3284 & 51.1 & 3232 & 8200 \\
\hline Xinjiang & 42635 & 30.2 & 42605 & 455480 \\
\hline National Total & 469346 & 4329 & 465016 & 1184800 \\
\hline
\end{tabular}

\#The data from the "The Second National Survey of Pollution Sources of China";

${ }^{*}$ No data available 
Table S14 Emission of DBP and DEHP released from plastic mulching film into air and soil compartments in provinces of China.

\begin{tabular}{|c|c|c|c|c|}
\hline \multirow{2}{*}{ Province } & \multicolumn{2}{|c|}{ DBP emission $(\mathrm{kg})$} & \multicolumn{2}{|c|}{ DEHP emission (kg) } \\
\hline & Air & Soil & Air & Soil \\
\hline Beijing & 6.40 & 19.5 & 14.4 & 43.9 \\
\hline Tianjin & 10.3 & 25.8 & 23.0 & 58.0 \\
\hline Hebei & 168 & 473 & 378 & 1064 \\
\hline Shanxi & 86.7 & 292 & 195 & 656 \\
\hline Inner Mongolia & 813 & 662 & 1826 & 1488 \\
\hline Liaoning & 397 & 253 & 892 & 569 \\
\hline Jilin & 313 & 174 & 703 & 392 \\
\hline Heilongjiang & 327 & 178 & 734 & 400 \\
\hline Shanghai & 3.89 & 32.9 & 8.75 & 73.9 \\
\hline Jiangsu & 46.1 & 392 & 104 & 881 \\
\hline Zhejiang & 29.7 & 213 & 66.6 & 479 \\
\hline Anhui & 44.1 & 552 & 99.2 & 1240 \\
\hline Fujian & 32.7 & 201 & 73.4 & 453 \\
\hline Jiangxi & 34.0 & 259 & 76.4 & 582 \\
\hline Shandong & 312 & 952 & 702 & 2138 \\
\hline Henan & 200 & 568 & 449 & 1276 \\
\hline Hubei & 38.5 & 422 & 86.5 & 948 \\
\hline Hunan & 58.0 & 492 & 130 & 1106 \\
\hline Guangdong & 27.4 & 232 & 61.5 & 522 \\
\hline Guangxi & 37.2 & 316 & 83.6 & 710 \\
\hline Hainan & 17.1 & 145 & 38.3 & 325 \\
\hline Chongqing & 67.4 & 210 & 151 & 473 \\
\hline Sichuan & 249 & 641 & 559 & 1440 \\
\hline Guizhou & 87.2 & 262 & 196 & 588 \\
\hline Yunnan & 263 & 801 & 591 & 1799 \\
\hline Tibet & 4.04 & 12.3 & 9.08 & 27.7 \\
\hline Shaanxi & 234 & 149 & 525 & 335 \\
\hline Gansu & 1135 & 741 & 2550 & 1664 \\
\hline Qinghai & 70.1 & 47.6 & 158 & 107 \\
\hline Ningxia & 121 & 77.4 & 272 & 174 \\
\hline Xinjiang & 2298 & 1465 & 5164 & 3291 \\
\hline
\end{tabular}


Table S15 Emission of DBP and DEHP released from greenhouse film into inside and outside greenhouse in provinces of China.

\begin{tabular}{|c|c|c|c|c|}
\hline \multirow{2}{*}{ Province } & \multicolumn{2}{|c|}{ DBP emission (kg) } & \multicolumn{2}{|c|}{ DEHP emission $(\mathrm{kg})$} \\
\hline & Inside & Outside & Inside & Outside \\
\hline Beijing & 18.4 & 17.7 & 75.7 & 73.2 \\
\hline Tianjin & 19.8 & 19.1 & 81.7 & 78.9 \\
\hline Hebei & 184 & 178 & 758 & 735 \\
\hline Shanxi & 50.8 & 48.6 & 209 & 201 \\
\hline Inner Mongolia & 46.7 & 44.2 & 193 & 182 \\
\hline Liaoning & 242 & 231 & 999 & 951 \\
\hline Jilin & 86.4 & 81.6 & 356 & 337 \\
\hline Heilongjiang & 136 & 128 & 563 & 528 \\
\hline Shanghai & 32.7 & 31.9 & 135 & 132 \\
\hline Jiangsu & 193 & 188 & 797 & 777 \\
\hline Zhejiang & 107 & 105 & 442 & 432 \\
\hline Anhui & 150 & 146 & 619 & 604 \\
\hline Fujian & 83.6 & 82.6 & 345 & 341 \\
\hline Jiangxi & 55.8 & 54.8 & 230 & 226 \\
\hline Shandong & 477 & 463 & 1968 & 1911 \\
\hline Henan & 232 & 226 & 958 & 934 \\
\hline Hubei & 77.9 & 76.1 & 321 & 314 \\
\hline Hunan & 78.8 & 77.0 & 325 & 317 \\
\hline Guangdong & 52.4 & 51.9 & 216 & 214 \\
\hline Guangxi & 31.2 & 30.9 & 128 & 127 \\
\hline Hainan & 30.0 & 29.9 & 124 & 123 \\
\hline Chongqing & 57.2 & 56.2 & 236 & 232 \\
\hline Sichuan & 110 & 108 & 455 & 443 \\
\hline Guizhou & 53.2 & 51.5 & 219 & 213 \\
\hline Yunnan & 66.0 & 64.1 & 272 & 264 \\
\hline Tibet & 1.09 & 1.04 & 4.50 & 4.29 \\
\hline Shaanxi & 59.7 & 58.0 & 246 & 239 \\
\hline Gansu & 178 & 169 & 735 & 697 \\
\hline Qinghai & 4.81 & 4.53 & 19.8 & 18.7 \\
\hline Ningxia & 9.75 & 9.33 & 40.2 & 38.5 \\
\hline Xinjiang & 92.7 & 87.9 & 382 & 362 \\
\hline
\end{tabular}


Table S16 Predicted concentrations of DBP and DEHP released from mulching film in China.

\begin{tabular}{|c|c|c|c|c|c|c|}
\hline \multirow[b]{2}{*}{ Province } & \multicolumn{3}{|c|}{ DBP } & \multicolumn{3}{|c|}{ DEHP } \\
\hline & $\begin{array}{c}\text { Air } \\
\left(\mathrm{ng} / \mathrm{m}^{3}\right)\end{array}$ & $\begin{array}{c}\text { Soil } \\
(\mathrm{mg} / \mathrm{kg})\end{array}$ & $\begin{array}{c}\text { Crop } \\
(\mathrm{mg} / \mathrm{kg})\end{array}$ & $\begin{array}{c}\text { Air } \\
\left(\mathrm{ng} / \mathrm{m}^{3}\right)\end{array}$ & $\begin{array}{c}\text { Soil } \\
(\mathrm{mg} / \mathrm{kg})\end{array}$ & $\begin{array}{c}\text { Crop } \\
(\mathrm{mg} / \mathrm{kg})\end{array}$ \\
\hline Beijing & $8.73 \times 10^{-4}$ & 0.09 & $5.78 \times 10^{-18}$ & $1.60 \times 10^{-3}$ & 0.20 & $9.73 \times 10^{-16}$ \\
\hline Tianjin & $2.06 \times 10^{-3}$ & 0.02 & $1.37 \times 10^{-17}$ & $3.75 \times 10^{-3}$ & 0.05 & $2.28 \times 10^{-15}$ \\
\hline Hebei & $1.94 \times 10^{-3}$ & 0.02 & $1.34 \times 10^{-17}$ & $3.57 \times 10^{-3}$ & 0.05 & $2.26 \times 10^{-15}$ \\
\hline Shanxi & $1.58 \times 10^{-3}$ & 0.03 & $9.13 \times 10^{-18}$ & $2.90 \times 10^{-3}$ & 0.06 & $1.53 \times 10^{-15}$ \\
\hline $\begin{array}{c}\text { Inner } \\
\text { Mongolia }\end{array}$ & $1.82 \times 10^{-3}$ & 0.04 & $8.65 \times 10^{-18}$ & $3.30 \times 10^{-3}$ & 0.09 & $1.41 \times 10^{-15}$ \\
\hline Liaoning & $6.43 \times 10^{-3}$ & 0.04 & $3.32 \times 10^{-17}$ & $1.16 \times 10^{-2}$ & 0.12 & $5.42 \times 10^{-15}$ \\
\hline Jilin & $4.61 \times 10^{-3}$ & 0.04 & $2.11 \times 10^{-17}$ & $8.30 \times 10^{-3}$ & 0.10 & $3.40 \times 10^{-15}$ \\
\hline Heilongjiang & $2.26 \times 10^{-3}$ & 0.03 & $9.38 \times 10^{-18}$ & $4.06 \times 10^{-3}$ & 0.07 & $1.49 \times 10^{-15}$ \\
\hline Shanghai & $1.68 \times 10^{-3}$ & 0.10 & $1.32 \times 10^{-17}$ & $3.14 \times 10^{-3}$ & 0.30 & $2.30 \times 10^{-15}$ \\
\hline Jiangsu & $1.30 \times 10^{-3}$ & 0.03 & $9.87 \times 10^{-18}$ & $2.46 \times 10^{-3}$ & 0.10 & $1.73 \times 10^{-15}$ \\
\hline Zhejiang & $7.50 \times 10^{-4}$ & 0.06 & $6.07 \times 10^{-18}$ & $1.39 \times 10^{-3}$ & 0.19 & $1.05 \times 10^{-15}$ \\
\hline Anhui & $9.35 \times 10^{-4}$ & 0.07 & $7.15 \times 10^{-18}$ & $1.83 \times 10^{-3}$ & 0.21 & $1.30 \times 10^{-15}$ \\
\hline Fujian & $5.63 \times 10^{-4}$ & 0.05 & $5.24 \times 10^{-18}$ & $1.02 \times 10^{-3}$ & 0.12 & $8.97 \times 10^{-16}$ \\
\hline Jiangxi & $4.94 \times 10^{-4}$ & 0.09 & $4.18 \times 10^{-18}$ & $9.20 \times 10^{-4}$ & 0.28 & $7.28 \times 10^{-16}$ \\
\hline Shandong & $4.22 \times 10^{-3}$ & 0.02 & $3.01 \times 10^{-17}$ & $7.82 \times 10^{-3}$ & 0.06 & $5.16 \times 10^{-15}$ \\
\hline Henan & $2.28 \times 10^{-3}$ & 0.02 & $1.72 \times 10^{-17}$ & $4.22 \times 10^{-3}$ & 0.07 & $2.95 \times 10^{-15}$ \\
\hline Hubei & $5.93 \times 10^{-4}$ & 0.06 & $4.58 \times 10^{-18}$ & $1.14 \times 10^{-3}$ & 0.17 & $8.16 \times 10^{-16}$ \\
\hline Hunan & $7.37 \times 10^{-4}$ & 0.03 & $5.80 \times 10^{-18}$ & $1.37 \times 10^{-3}$ & 0.10 & $1.00 \times 10^{-15}$ \\
\hline Guangdong & $3.02 \times 10^{-4}$ & 0.06 & $2.93 \times 10^{-18}$ & $5.54 \times 10^{-4}$ & 0.15 & $5.09 \times 10^{-16}$ \\
\hline Guangxi & $3.16 \times 10^{-4}$ & 0.02 & $3.04 \times 10^{-18}$ & $5.77 \times 10^{-4}$ & 0.05 & $5.25 \times 10^{-16}$ \\
\hline Hainan & $8.43 \times 10^{-4}$ & 0.09 & $9.20 \times 10^{-18}$ & $1.57 \times 10^{-3}$ & 0.27 & $1.64 \times 10^{-15}$ \\
\hline Chongqing & $1.29 \times 10^{-3}$ & 0.03 & $1.10 \times 10^{-17}$ & $2.39 \times 10^{-3}$ & 0.10 & $1.91 \times 10^{-15}$ \\
\hline Sichuan & $9.85 \times 10^{-4}$ & 0.03 & $7.35 \times 10^{-18}$ & $1.79 \times 10^{-3}$ & 0.07 & $1.24 \times 10^{-15}$ \\
\hline Guizhou & $1.04 \times 10^{-3}$ & 0.04 & $7.26 \times 10^{-18}$ & $1.89 \times 10^{-3}$ & 0.10 & $1.21 \times 10^{-15}$ \\
\hline Yunnan & $1.40 \times 10^{-3}$ & 0.04 & $9.96 \times 10^{-18}$ & $2.54 \times 10^{-3}$ & 0.09 & $1.67 \times 10^{-15}$ \\
\hline Tibet & $1.02 \times 10^{-5}$ & 0.28 & $5.41 \times 10^{-20}$ & $1.89 \times 10^{-5}$ & 0.86 & $9.07 \times 10^{-18}$ \\
\hline Shaanxi & $1.73 \times 10^{-3}$ & 0.01 & $1.23 \times 10^{-17}$ & $3.13 \times 10^{-3}$ & 0.03 & $2.05 \times 10^{-15}$ \\
\hline Gansu & $6.47 \times 10^{-3}$ & 0.03 & $3.13 \times 10^{-17}$ & $1.17 \times 10^{-2}$ & 0.08 & $5.08 \times 10^{-15}$ \\
\hline Qinghai & $2.82 \times 10^{-4}$ & 0.04 & $1.25 \times 10^{-18}$ & $5.11 \times 10^{-4}$ & 0.10 & $2.01 \times 10^{-16}$ \\
\hline Ningxia & $3.84 \times 10^{-3}$ & 0.02 & $2.17 \times 10^{-17}$ & $6.93 \times 10^{-3}$ & 0.03 & $3.54 \times 10^{-15}$ \\
\hline Xinjiang & $3.51 \times 10^{-3}$ & 0.02 & $1.72 \times 10^{-17}$ & $6.36 \times 10^{-3}$ & 0.06 & $2.80 \times 10^{-15}$ \\
\hline
\end{tabular}


Table S17 Predicted concentrations of DBP and DEHP released from greenhouse film in China.

\begin{tabular}{|c|c|c|c|c|c|c|}
\hline \multirow[b]{2}{*}{ Province } & \multicolumn{3}{|c|}{ DBP } & \multicolumn{3}{|c|}{ DEHP } \\
\hline & $\begin{array}{c}\text { Air } \\
\left(\mathrm{ng} / \mathrm{m}^{3}\right)\end{array}$ & $\begin{array}{c}\text { Soil } \\
(\mathrm{mg} / \mathrm{kg})\end{array}$ & $\begin{array}{c}\text { Crop } \\
(\mathrm{mg} / \mathrm{kg})\end{array}$ & $\begin{array}{c}\text { Air } \\
\left(\mathrm{ng} / \mathrm{m}^{3}\right)\end{array}$ & $\begin{array}{c}\text { Soil } \\
(\mathrm{mg} / \mathrm{kg})\end{array}$ & $\begin{array}{c}\text { Crop } \\
(\mathrm{mg} / \mathrm{kg})\end{array}$ \\
\hline Beijing & 12.4 & $6.35 \times 10^{-4}$ & $1.37 \times 10^{-2}$ & 4.63 & $4.25 \times 10^{-4}$ & $4.78 \times 10^{-2}$ \\
\hline Tianjin & 5.02 & $2.58 \times 10^{-4}$ & $5.57 \times 10^{-3}$ & 2.71 & $2.48 \times 10^{-4}$ & $2.79 \times 10^{-2}$ \\
\hline Hebei & 3.80 & $1.95 \times 10^{-4}$ & $4.21 \times 10^{-3}$ & 1.44 & $1.31 \times 10^{-4}$ & $1.48 \times 10^{-2}$ \\
\hline Shanxi & 3.98 & $2.04 \times 10^{-4}$ & $4.41 \times 10^{-3}$ & 2.28 & $2.08 \times 10^{-4}$ & $2.36 \times 10^{-2}$ \\
\hline $\begin{array}{c}\text { Inner } \\
\text { Mongolia }\end{array}$ & 3.84 & $1.98 \times 10^{-4}$ & $4.26 \times 10^{-3}$ & 1.63 & $1.50 \times 10^{-4}$ & $1.68 \times 10^{-2}$ \\
\hline Liaoning & 8.92 & $4.58 \times 10^{-4}$ & $9.89 \times 10^{-3}$ & 4.93 & $4.52 \times 10^{-4}$ & $5.10 \times 10^{-2}$ \\
\hline Jilin & 12.1 & $6.21 \times 10^{-4}$ & $1.34 \times 10^{-2}$ & 8.31 & $7.64 \times 10^{-4}$ & $8.59 \times 10^{-2}$ \\
\hline Heilongjiang & 8.55 & $4.40 \times 10^{-4}$ & $9.49 \times 10^{-3}$ & 5.87 & $5.40 \times 10^{-4}$ & $6.06 \times 10^{-2}$ \\
\hline Shanghai & 13.2 & $6.78 \times 10^{-4}$ & $1.46 \times 10^{-2}$ & 4.47 & $4.10 \times 10^{-4}$ & $4.62 \times 10^{-2}$ \\
\hline Jiangsu & 4.23 & $2.18 \times 10^{-4}$ & $4.70 \times 10^{-3}$ & 1.40 & $1.29 \times 10^{-4}$ & $1.45 \times 10^{-2}$ \\
\hline Zhejiang & 8.66 & $4.45 \times 10^{-4}$ & $9.60 \times 10^{-3}$ & 2.32 & $2.13 \times 10^{-4}$ & $2.39 \times 10^{-2}$ \\
\hline Anhui & 6.63 & $3.40 \times 10^{-4}$ & $7.35 \times 10^{-3}$ & 2.87 & $2.63 \times 10^{-4}$ & $2.96 \times 10^{-2}$ \\
\hline Fujian & 9.88 & $5.08 \times 10^{-4}$ & $1.10 \times 10^{-2}$ & 2.46 & $2.26 \times 10^{-4}$ & $2.54 \times 10^{-2}$ \\
\hline Jiangxi & 7.86 & $4.04 \times 10^{-4}$ & $8.71 \times 10^{-3}$ & 1.59 & $1.46 \times 10^{-4}$ & $1.64 \times 10^{-2}$ \\
\hline Shandong & 4.10 & $2.10 \times 10^{-4}$ & $4.55 \times 10^{-3}$ & 2.17 & $1.98 \times 10^{-4}$ & $2.24 \times 10^{-2}$ \\
\hline Henan & 4.02 & $2.06 \times 10^{-4}$ & $4.46 \times 10^{-3}$ & 1.25 & $1.15 \times 10^{-4}$ & $1.29 \times 10^{-2}$ \\
\hline Hubei & 3.70 & $1.90 \times 10^{-4}$ & $4.10 \times 10^{-3}$ & 0.85 & $7.84 \times 10^{-5}$ & $8.83 \times 10^{-3}$ \\
\hline Hunan & 3.50 & $1.80 \times 10^{-4}$ & $3.88 \times 10^{-3}$ & 0.89 & $8.20 \times 10^{-5}$ & $9.21 \times 10^{-3}$ \\
\hline Guangdong & 4.07 & $2.09 \times 10^{-4}$ & $4.51 \times 10^{-3}$ & 0.71 & $6.50 \times 10^{-5}$ & $7.31 \times 10^{-3}$ \\
\hline Guangxi & 1.99 & $1.02 \times 10^{-4}$ & $2.21 \times 10^{-3}$ & 0.40 & $3.70 \times 10^{-5}$ & $4.16 \times 10^{-3}$ \\
\hline Hainan & 11.1 & $5.72 \times 10^{-4}$ & $1.23 \times 10^{-2}$ & 2.30 & $2.10 \times 10^{-4}$ & $2.37 \times 10^{-2}$ \\
\hline Chongqing & 4.63 & $2.38 \times 10^{-4}$ & $5.13 \times 10^{-3}$ & 1.27 & $1.16 \times 10^{-4}$ & $1.31 \times 10^{-2}$ \\
\hline Sichuan & 4.17 & $2.14 \times 10^{-4}$ & $4.62 \times 10^{-3}$ & 1.08 & $9.90 \times 10^{-5}$ & $1.11 \times 10^{-2}$ \\
\hline Guizhou & 4.12 & $2.12 \times 10^{-4}$ & $4.56 \times 10^{-3}$ & 0.98 & $9.00 \times 10^{-5}$ & $1.01 \times 10^{-2}$ \\
\hline Yunnan & 4.56 & $2.35 \times 10^{-4}$ & $5.06 \times 10^{-3}$ & 1.31 & $1.20 \times 10^{-4}$ & $1.35 \times 10^{-2}$ \\
\hline Tibet & 4.35 & $2.24 \times 10^{-4}$ & $4.83 \times 10^{-3}$ & 0.65 & $5.98 \times 10^{-5}$ & $6.72 \times 10^{-3}$ \\
\hline Shaanxi & 3.28 & $1.68 \times 10^{-4}$ & $3.64 \times 10^{-3}$ & 1.34 & $1.23 \times 10^{-4}$ & $1.39 \times 10^{-2}$ \\
\hline Gansu & 6.25 & $3.21 \times 10^{-4}$ & $6.93 \times 10^{-3}$ & 4.84 & $4.43 \times 10^{-4}$ & $5.00 \times 10^{-2}$ \\
\hline Qinghai & 4.57 & $2.35 \times 10^{-4}$ & $5.07 \times 10^{-3}$ & 1.33 & $1.22 \times 10^{-4}$ & $1.38 \times 10^{-2}$ \\
\hline Ningxia & 2.78 & $1.43 \times 10^{-4}$ & $3.09 \times 10^{-3}$ & 0.75 & $6.83 \times 10^{-5}$ & $7.72 \times 10^{-3}$ \\
\hline Xinjiang & 4.02 & $2.07 \times 10^{-4}$ & $4.46 \times 10^{-3}$ & 1.93 & $1.76 \times 10^{-4}$ & $1.99 \times 10^{-2}$ \\
\hline
\end{tabular}


Table S18 Comparison between predicted and observed DBP and DEHP concentrations in mulching film soil $\left(\mathrm{mg} \cdot \mathrm{kg}^{-1}\right)$.

\begin{tabular}{|c|c|c|c|c|c|}
\hline \multirow{2}{*}{ Province } & \multicolumn{2}{|c|}{ DBP } & \multicolumn{2}{|c|}{ DEHP } & \multirow{2}{*}{ Reference } \\
\hline & Predicted & Observed & Predicted & Observed & \\
\hline Beijing & 0.088 & 0.440 & 0.203 & 0.380 & Li et al., 2016 \\
\hline Tianjin & 0.022 & 0.126 & 0.051 & 0.820 & Ren et al., 2018 \\
\hline Hebei & 0.022 & 0.045 & 0.054 & 0.143 & Zhang et al., 2015 \\
\hline Heilongjiang & 0.039 & 0.159 & 0.118 & 0.222 & Wang et al., 2017 \\
\hline Shanghai & 0.030 & 0.095 & 0.069 & 0.546 & Sun et al., 2016 \\
\hline Jiangsu & 0.100 & 0.450 & 0.296 & 0.984 & Ma et al., 2015 \\
\hline Zhejiang & 0.035 & 0.020 & 0.097 & 0.108 & Fei et al., 2010 \\
\hline Anhui & 0.062 & 0.410 & 0.190 & 1.200 & Hu et al., 2003 \\
\hline Jiangxi & 0.075 & 0.058 & 0.211 & 0.087 & Xiong et al., 2008 \\
\hline Shandong & 0.085 & 0.413 & 0.279 & 1.224 & Yang et al., 2013 \\
\hline Henan & 0.023 & 0.260 & 0.058 & 0.540 & Hu et al., 2003 \\
\hline Guangdong & 0.024 & 0.342 & 0.067 & 1.755 & Zeng et al., 2008 \\
\hline Chongqing & 0.062 & 0.254 & 0.151 & 0.118 & He et al., 2017 \\
\hline Guizhou & 0.030 & 0.771 & 0.100 & 1.691 & Zhang et al., 2018 \\
\hline Shaanxi & 0.040 & 0.314 & 0.098 & 0.163 & Xu et al., 2014 \\
\hline Xinjiang & 0.020 & 0.244 & 0.055 & 1.999 & Li YL, 2014 \\
\hline
\end{tabular}


Table S19 Measured concentration of DBP and DEHP in soil and vegetables of greenhouse film area.

\begin{tabular}{cccccccc}
\hline Province & \multicolumn{3}{c}{ Target compound in soil (mg/kg) } & & \multicolumn{3}{c}{ Target compound in vegetable (mg/kg) } \\
\cline { 2 - 3 } \cline { 7 - 8 } & DBP & DEHP & Reference & & DBP & DEHP & Reference \\
\hline Beijing & 0.425 & 0.369 & Chen et al., 2016 & & 0.50 & 0.55 & Li et al., 2016 \\
Tianjin & 0.068 & 0.471 & Kong et al., 2012 & & - & - & - \\
Hebei & 0.162 & 0.101 & Lei et al.,2014 & & - & - & - \\
Liaoning & 0.165 & 0.046 & Chen et al., 2016a & & - & - & - \\
Shanghai & 0.10 & 0.45 & Sun et al., 2018 & & 0.72 & 2.25 & Sun et al., 2018 \\
Jiangsu & 0.075 & 0.028 & Feng et al.,2017 & & 0.63 & 1.09 & Wang et al., 2015 \\
Zhejiang & - & - & - & & 0.26 & 2.25 & Liu et al.,2010 \\
Shandong & 0.435 & 0.671 & Zheng et al.,2016 & & 0.40 & 0.46 & Zheng et al.,2016 \\
Guangdong & 0.146 & 0.410 & Zhang et al.,2009 & & 0.20 & 0.30 & Zhang et al., 2009 \\
Shaanxi & 0.123 & 0.253 & Xu et al.,2014 & & - & - & - \\
Xinjiang & 0.456 & 0.629 & Li et al.,2018 & & - & - & - \\
\hline
\end{tabular}




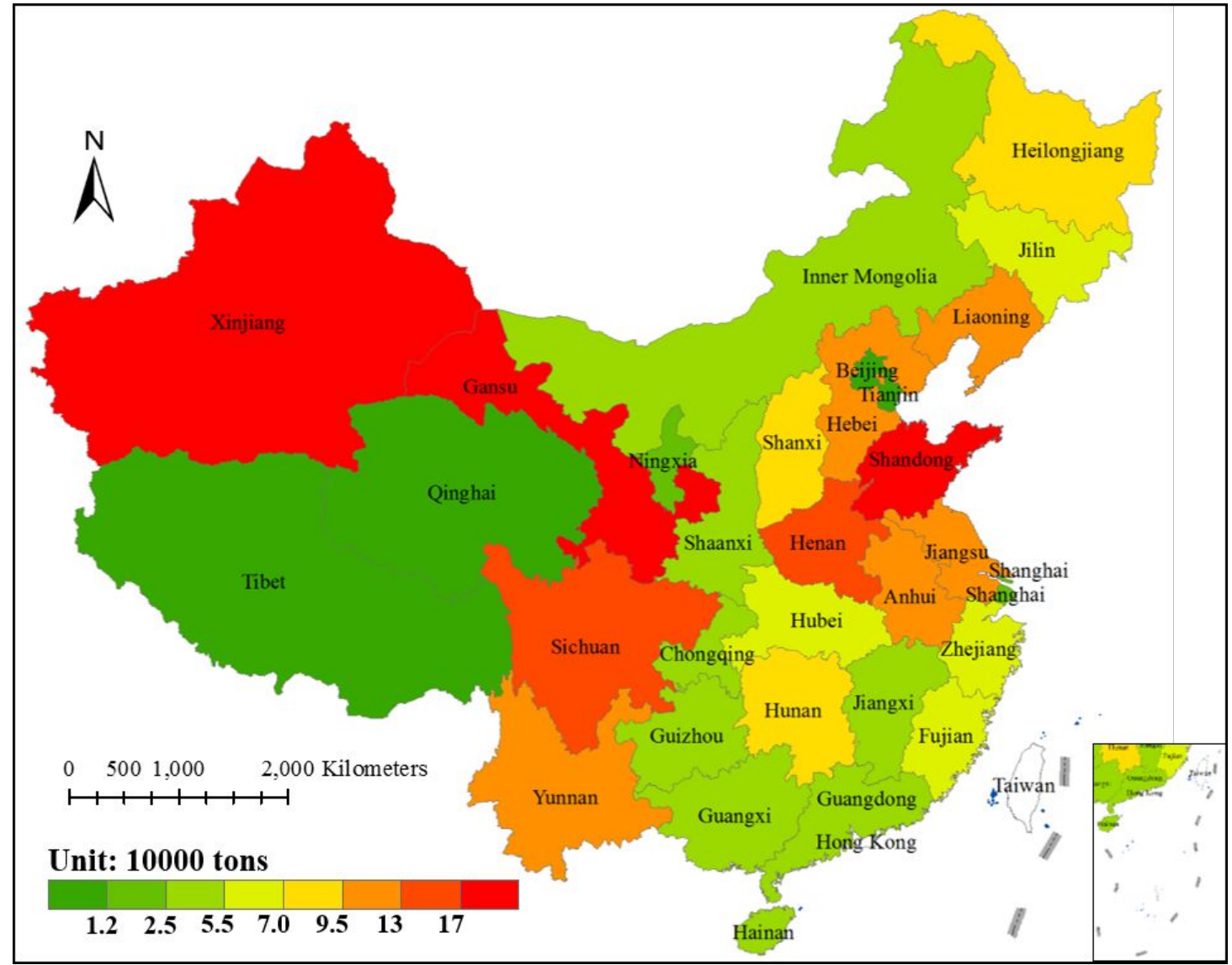

Figure S1 Distribution of agricultural film usage in China in 2017. 


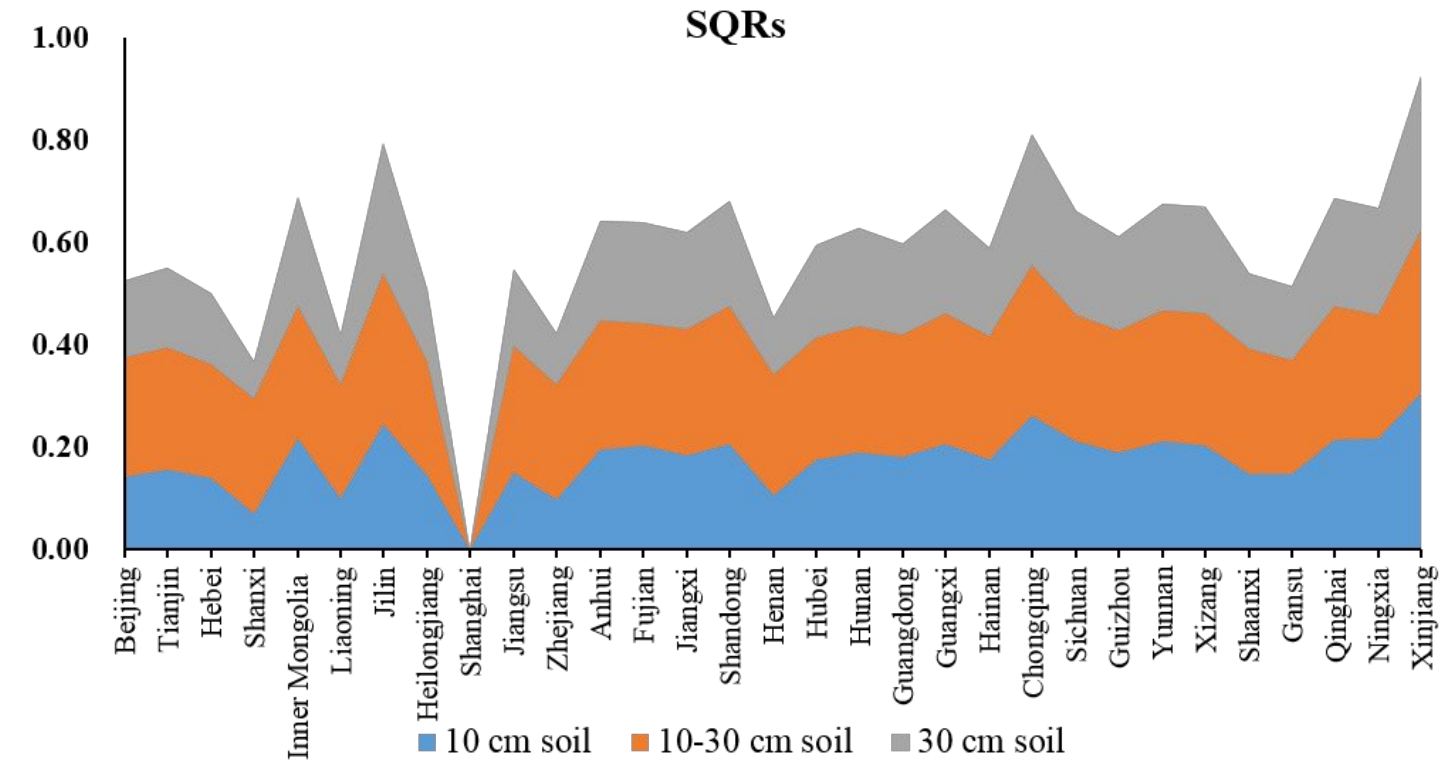

Figure S2 The uncertainty simulation results of residual agricultural film distribution in different soil layers. 


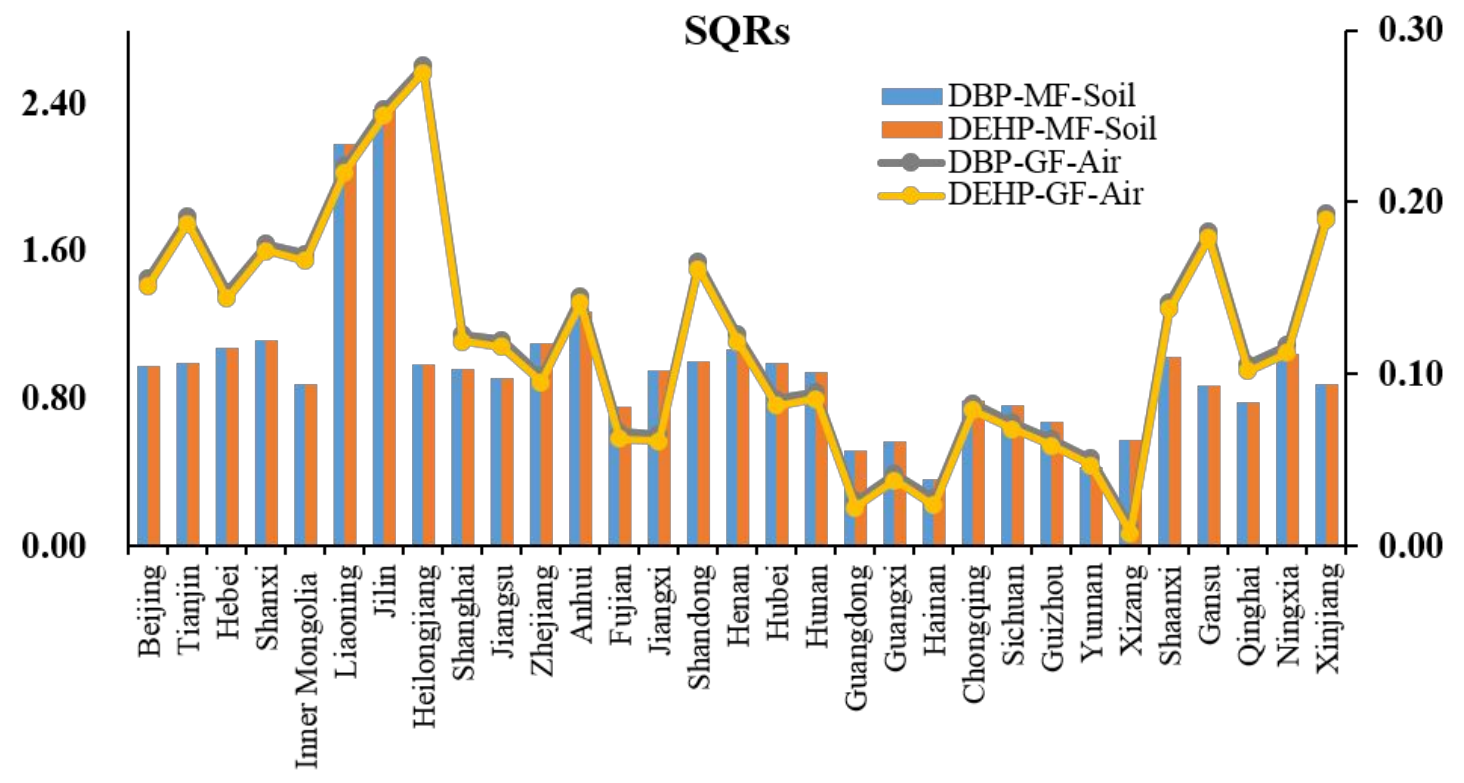

Figure S3 The uncertainty simulation results in terms of predicted concentrations of multimedia model. The left coordinate is for soil compartment, and the right coordinate is for air compartment. 


\section{Greenhouse Film}
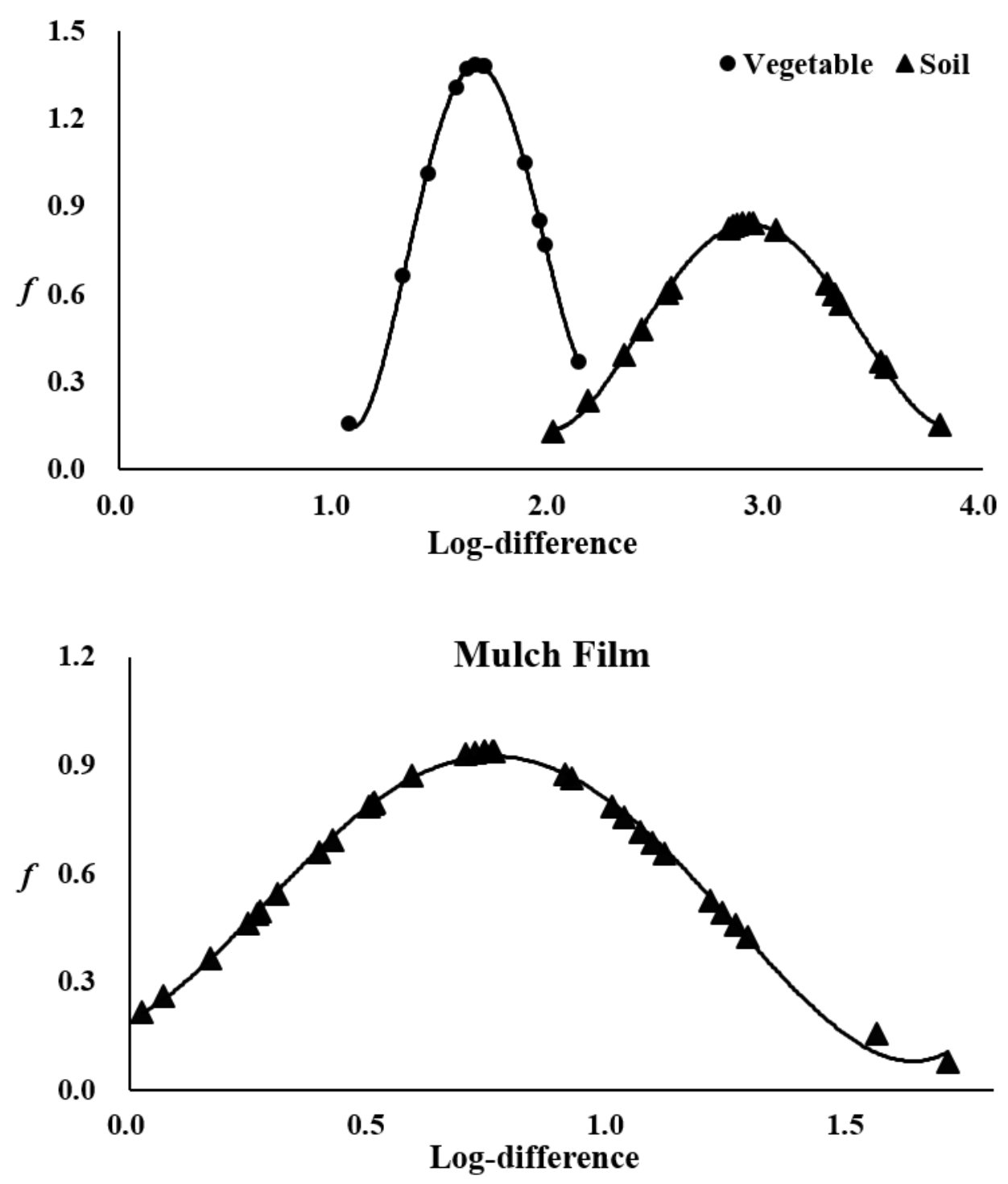

Figure S4 Normal distribution of magnitude differences between simulations and observations. 


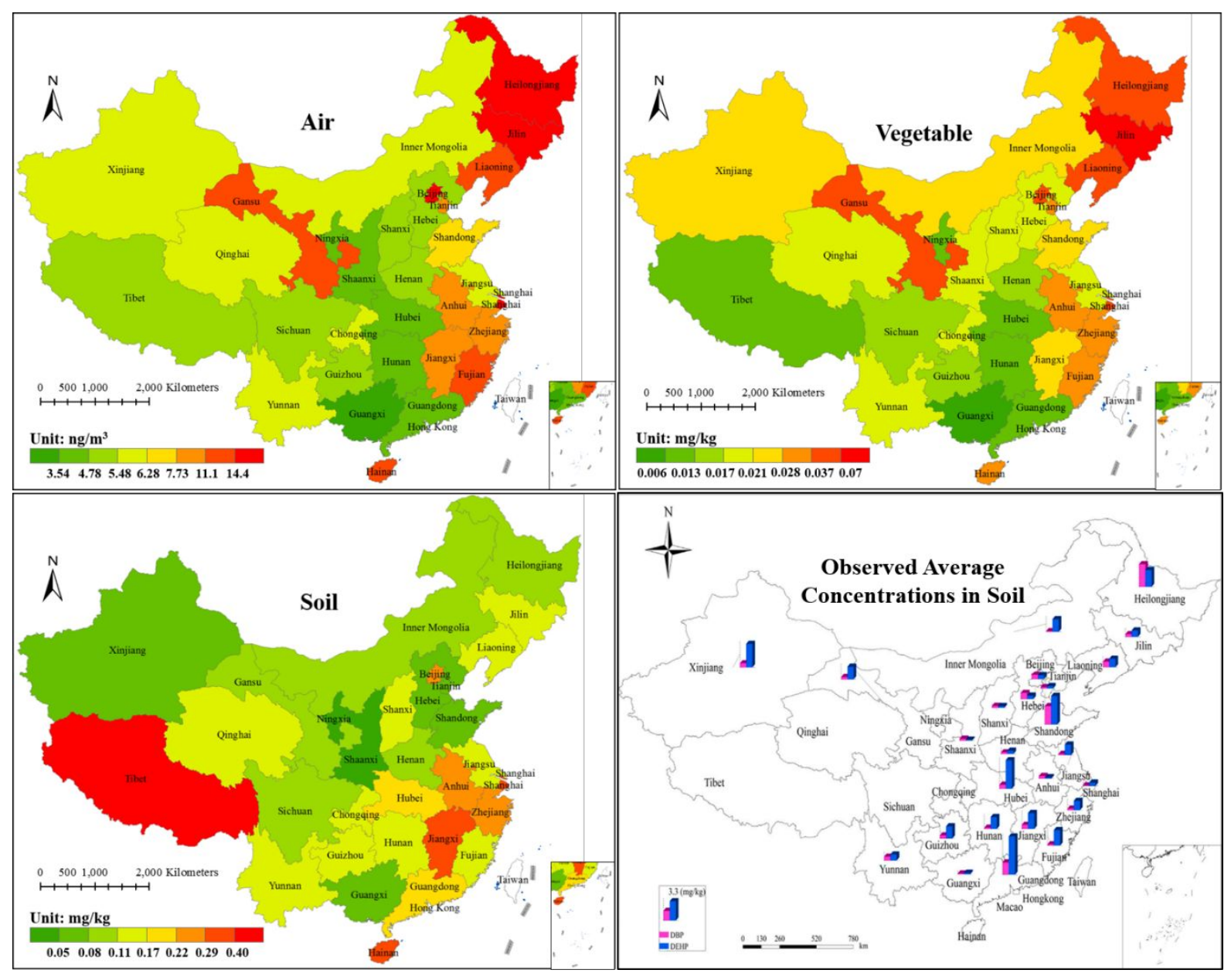

Figure S5 $\sum$ PAEs in air, soil and vegetable from agricultural film. The fourth map collected from reference (Lv et al., 2018). 


\section{REFERENCES}

1. An, Q.; Jin, W.; Li, Y.; Xu, R., Effect of PAEs plasticizers on soil-crop system. Acta Pedologica Sinica 1999, 36, (01), 118-126.

2. Chen, W.; Chi, C.; Zhou, C.; Xia, M.; Ronda, C.; Shen, X., Analysis of the influencing factors of PAEs volatilization from typical plastic products. J Environ Sci 2018, 66, 61-70.

3. DRS, China Rural Statistical Yearbook. In Department of rural social and economic investigation, Department of rural social and economic investigation, National Bureau of Statistics, Ed. China Statistics Press 2018.

4. EC, European Commision, Institute for Health and Consumer Protection : EUR 23384 EN/2 Summary risk assessment report on bis(2-ethylhexyl)phthalate (DEHP). Luxembourg 2008, 80 .

5. Fei, Y.; Chen, J.; Ni, X.; Wu, H.; Wu, j., Spatial distribution of phthalate esters in soil of Huzhou area. Journal of Agricultural Resources and Environment 2010, 27, (2), 83-86.

6. He, M. J.; Yang, T.; Yang, Z. H.; Zhou, H.; Wei, S. Q., Current state, distribution, and sources of phthalate esters and organophosphate esters in soils of the Three Gorges Reservoir region, China. Arch Environ Contam Toxicol 2017.

7. $\mathrm{Hu}, \mathrm{X} .-\mathrm{y} . ;$ Wen, B.; Shan, X.-q., Survey of phthalate pollution in arable soils in China. J Environ Monitor 2003, 5, (4), 649-653.

8. Li, C.; Chen, J.; Wang, J.; Han, P.; Luan, Y.; Ma, X.; Lu, A., Phthalate esters in soil, plastic film, and vegetable from greenhouse vegetable production bases in Beijing, China: Concentrations, sources, and risk assessment. Sci Total Environ 2016, 568, 1037-1043.

9. Li, M., Effect of plastic film mulching with different time of uncovering film on yield of Spring Potato. Shanghai Agricultural Science and Technology 2007, (4), 9394.

10. Li, Y., Investigation and evaluation on soil pollution for phthalate esters of JiaMusi City. Arid Environmental Monitoring 2014, 28, (1), 22-24.

11. Liang, D. W.; Zhang, T.; Fang, H. H.; He, J., Phthalates biodegradation in the environment. Appl Microbiol Biotechnol 2008, 80, (2), 183-98.

12. Liu, S.; Dou, S.; Hou, Y.; Zheng, H.; Huang, Z., Relationship between area of straw returning to the field and content of soil organic carbon in China. Journal of Jilin Agricultural University 2016, 28, (6), 723-732.

13. Liu, W.; Zhang, Z.; Zhu, Q.; Shen, C.; Wang, J., Distribution characteristics of phalatic esters in soil and plants at e-waste recycling sites in Taizhou of Zhejiang, China. Chinese Journal of Applied Ecology 2010, 21, (02), 489-494.

14. Lv, H. X.; Mo, C. H.; Zhao, H. M.; Xiang, L.; Katsoyiannis, A.; Li, Y. W.; Cai, Q. Y.; Wong, M. H., Soil contamination and sources of phthalates and its health risk in China: A review. Environ Res 2018, 164, 417-429.

15. Ma, T. T.; Wu, L. H.; Chen, L.; Zhang, H. B.; Teng, Y.; Luo, Y. M., Phthalate esters contamination in soils and vegetables of plastic film greenhouses of suburb Nanjing, China and the potential human health risk. Environ Sci Pollut R 2015, 22, (16), 12018-12028.

16. Mackay, D., Multimedia environmental models : the fugacity approach. 2nd ed. ed.; Lewis Publishers: Boca Raton ; London, 2001.

17. Madsen, P. L.; Thyme, J. B.; Henriksen, K.; Møldrup, P.; Roslev, P., Kinetics of di-(2-ethylhexyl) phthalate mineralization in sludge-amended soil. Environ Sci 
Technol 1999, 33, (15), 2601-2606.

18. Mo, C.H.; Cai, Q.Y.; Tang, S.R.; Zeng, Q.Y.; Wu, Q.-T., Polycyclic aromatic hydrocarbons and phthalic acid esters in vegetables from nine farms of the Pearl River Delta, South China. Arch Environ Con Tox 2009, 56, (2), 181-189.

19. MWR, China water statistical yearbook. In China, Ministry of Water Resources of the People's Republic of China, Ed. China Water\&Power Press: Beijing, 2018. 20. NBSC, China Rural Statistical Yearbook. In China, National Bureau of Statistics of China, Ed. 2018.

21. Net, S.; Sempéré, R.; Delmont, A.; Paluselli, A.; Ouddane, B., Occurrence, fate, behavior and ecotoxicological state of phthalates in different environmental matrices. Environ Sci Technol 2015, 49, (7), 4019-4035.

22. Qin, L. Investigation of potato safety period of plastic film mulching in intensive agricultural region of North China. Chinese Academy of Agricultural Sciences, Beijing, 2018.

23. Ren, C.; Zhao, Z.; Liu, J.; Wang, Y.; Sun, H., Distribution and risk assessment of phthalic acid ester ( PAEs) in soil from a multi-waste recycling area. Environ Chem 2018, 37, (8), 1691-1698.

24. Sun, J.; Pan, L.; Tsang, D. C. W.; Li, Z.; Zhu, L.; Li, X., Phthalate esters and organochlorine pesticides in agricultural soils and vegetables from fast-growing regions: a case study from eastern China. Environ Sci Pollut $R$ 2018, 25, (1), 34-42. 25. Sun, J.; Pan, L.; Zhan, Y.; Lu, H.; Tsang, D. C. W.; Liu, W.; Wang, X.; Li, X.; Zhu, L., Contamination of phthalate esters, organochlorine pesticides and polybrominated diphenyl ethers in agricultural soils from the Yangtze River Delta of China. Sci Total Environ 2016, 544, 670-676.

26. Wang, H.; Liang, H.; Gao, D. W., Occurrence and risk assessment of phthalate esters (PAEs) in agricultural soils of the Sanjiang Plain, northeast China. Enviro Sci Pollut Res Int 2017, 24, (24), 1-10.

27. Wang, J.; Chen, G. C.; Christie, P.; Zhang, M. Y.; Luo, Y. M.; Teng, Y., Occurrence and risk assessment of phthalate esters (PAEs) in vegetables and soils of suburban plastic film greenhouses. Sci Total Environ 2015, 523, 129-137.

28. Wang, Y.; Wang, F.; Xiang, L. L.; Gu, C. G.; Redmile-Gordon, M.; Sheng, H. J.; Wang, Z. Q.; Fu, Y. H.; Bian, Y. R.; Jiang, X., Risk assessment of agricultural plastic films based on release kinetics of phthalate acid esters. Environ Sci Technol 2021, 55, (6), 3676-3685.

29. Wang, Y.; Zhan, W.; Ren, Q.; Cheng, S.; Wang, J.; Ma, X.; Zhang, C.; Wang, Y., Biodegradation of di-(2-ethylhexyl) phthalate by a newly isolated Gordonia sp. and its application in the remediation of contaminated soils. Sci Total Environ 2019, 689, 645-651.

30. Xiong, P.; Gong, X.; Deng, L., Analysis of PAE pollutants in farm soil and water samples in Nanchang city. Chemistry Bulletin 2008, 71, (8), 636-640.

31. Xu, X.; Wang, L.; Lu, X., Pollution of phthalic acid esters (PAEs) in vegetable soils in Xianyang Suburbs, Northwest China. Journal of Agro-Environment Science 2014, 33, (10), 1912-1919.

32. Yan, C., Application of mulch film and prevention of its residual pollution in China. Science Press 2015.

33. Yang, H.; Xie, W.; Liu, Q.; Liu, J.; Yu, H.; Lu, Z., Distribution of phthalate esters in topsoil: a case study in the Yellow River Delta, China. Environ Monit Assess 2013, 185, (10), 8489-8500.

34. Yang, J.; He, H., Effects of plastic film mulching time on growth and quality of 
flue cured tobacco in high altitude area Journal of Guizhou Meteorology 1997, 21, (6), 17-20.

35. Zeng, F.; Cui, K.; Xie, Z.; Wu, L.; Liu, M.; Sun, G.; Lin, Y.; Luo, D.; Zeng, Z., Phthalate esters (PAEs): emerging organic contaminants in agricultural soils in periurban areas around Guangzhou, China. Environ Pollut 2008, 156, (2), 425-434.

36. Zhang, M.S.; Li, M.Y.; Wang, J.I.; Wang, Q.J.; Luo, H.H.; He, Z.Z.; He, J.W.; Mo, C.H., Occurrence of phthalic acid esters (PAEs) in vegetable fields of Dongguan City. Guaangdong Agricultural Science 2009, (06), 173-176+181.

37. Zhang, Q. Q.; Ying, G. G.; Chen, Z. F.; Zhao, J. L.; Liu, Y. S., Basin-scale emission and multimedia fate of triclosan in whole. Environ Sci Pollut R 2015a, 22, (13), 10130-10143.

37. Zhang, Q. Q.; Ying, G. G.; Pan, C. G.; Liu, Y. S.; Zhao, J. L., Comprehensive evaluation of antibiotics emission and fate in the river basins of China: Source analysis, multimedia modeling, and linkage to bacterial resistance. Environ Sci Technol 2015, 49, (11), 6772-6782.

38. Zhang, Q. Q.; Zhao, J. L.; Ying, G. G.; Liu, Y. S.; Pan, C. G., Emission estimation and multimedia fate modeling of seven steroids at the river basin scale in China. Environ Sci Technol 2014, 48, (14), 7982-7992.

39. Zhang, X.; Li, Z.; Chen, Q.; Xu, L.; Sun, G.; Zhang, S.; Jiao, J., Effects of mulching duration on PAEs accumulation in soil and tobacco leaves in Zunyi, China. Chinese Journal of Applied Ecology 2018, 29, (10), 155-162.

40. Zhang, Y.; Liang, Q.; Gao, R.; Hou, H.; Tan, W.; He, X.; Zhang, H.; Yu, M.; Ma, L.; Xi, B., Contamination of phthalate esters (PAEs) in typical wastewater-irrigated agricultural soils in Hebei, North China. Plos One 2015, 10, (9), e0137998.

41. Zhao, X. Effects of different film-removing time on summer maize growth and yield. Northwest A\&F University, Shaanxi, 2015.

42. Zheng, S.-a.; Xue, Y.-h.; Li, X.-h.; Duan, Q.; Gao, S., Phthalate acid esters (PAEs) pollution in soils and agricultural products of vegetable greenhouses in Shouguang City, Shandong Province. Journal of Agro-Environment Science 2016, 35 , (3), 492-499.

43. Zhu, D.; Zou, S.; Zhou, C.; Liu, F.; Xie, H.; Lu, H., Phthalate from agricultural plastic film in the Karst Plateau area and its effect on film -covered soil. Rock and Mineral Analysis 2019, 38, (3), 297-304. 